\title{
Langmuir Turbulence in Coastal Zones: Structure and Length Scales
}

\author{
KALYAN SHRESTHA AND WiLliam ANDERSON \\ Mechanical Engineering Department, The University of Texas at Dallas, Richardson, Texas \\ JOSEPH KUEHL \\ Mechanical Engineering Department, University of Delaware, Newark, Delaware
}

(Manuscript received 3 April 2017, in final form 16 March 2018)

\begin{abstract}
Langmuir turbulence is a boundary layer oceanographic phenomenon of the upper layer that is relevant to mixing and vertical transport capacity. It is a manifestation of imposed aerodynamic stresses and the aggregate horizontal velocity profile due to orbital wave motion (the so-called Stokes profile), resulting in streamwise-elongated, counterrotating cells. The majority of previous research on Langmuir turbulence has focused on the open ocean. Here, we investigate the characteristics of coastal Langmuir turbulence by solving the grid-filtered Craik-Leibovich equations where the distinction between open and coastal conditions is a product of additional bottom boundary layer shear. Studies are elucidated by visualizing Langmuir cell vortices using isosurfaces of $Q$. We show that different environmental forcing conditions control the length scales of coastal Langmuir cells. We have identified regimes where increasing the Stokes drift velocity and decreasing surface wind stress both act to change the horizontal size of coastal Langmuir cells. Furthermore, wavenumber is also responsible in setting the horizontal extent $L_{s}$ of Langmuir cells. Along with that, wavenumber that is linked to the Stokes depth $\delta_{s}$ controls the vertical extent $L_{h}^{\text {ssv }}$ of small-scale vortices embedded within the upwelling limb, while the downwelling limb occupies the depth of the water column $H$ for any coastal surface wave forcing (i.e., $L_{h}^{d}=H$ and $L_{h}^{\text {ssv }} \sim \delta_{s}$ ). Additional simulations are included to demonstrate insensitivity to the grid resolution and aspect ratio.
\end{abstract}

\section{Introduction}

The upper region of the ocean is subjected to dramatic ambient forcing, which enhances and controls the turbulent exchanges of momentum, heat, and other quantities across the air-sea interface (Leibovich 1983; Thorpe 2004; Sullivan and Williams 2010). Among these forces are breaking and nonbreaking waves, where the latter interacts with wind stress to produce local recirculating motions called Langmuir circulations (LC), which was first studied by Langmuir (1938). LC can be observed on the sea surface as parallel streaks, coaligned with the prevailing wind direction, with lateral spacing varying between a few meters to hundreds of meters. These streaks are the product of surface convergence, where, by continuity, the water must sink, and the collective result is formation of counterrotating vortical structures. LC is characterized as a turbulent self-organization

\footnotetext{
Corresponding author: Kalyan Shrestha, kxs160530@utdallas. edu
}

due to the interaction of surface waves and winddriven shear instability in the surface mixed layer. Langmuir turbulence can be numerically modeled via solution of the momentum transport equations with an additional vortex forcing term, which modulates turbulent momentum transport in the lateral and vertical direction such that counterrotating LCs emerge; this transport equation term is referred to as the Craik-Leibovich (C-L), first proposed by Craik and Leibovich (1976). An improved second generation (CL-2) is more commonly used to model Langmuir turbulence (Leibovich 1977a,b). The vortex force $\mathbf{f}_{L}$ (also called C-L force) is included in the momentum transport equations and is the curl of the Stokes drift $\left[\mathbf{u}_{s}(z)\right]$ and ambient vorticity $[\tilde{\boldsymbol{\omega}}(\mathbf{x}, t)]$ ( $\ldots$ denotes a grid-filtered LES quantity), given by $\mathbf{f}_{L}(\mathbf{x}, t)=\mathbf{u}_{s}(z) \times \tilde{\boldsymbol{\omega}}(\mathbf{x}, t)$ (in this article, the streamwise, spanwise, and vertical direction correspond with the first, second, and third component of any vector, where the spatial vector is $\mathbf{x}=x \hat{\mathbf{i}}+y \hat{\mathbf{j}}+z \hat{\mathbf{k}} ;$ vectors are denoted with bold roman, e.g., $\mathbf{x}$, while tensors are 
denoted by bold sans serif, e.g., A). This vortex force represents an inviscid wave-induced instability of the unidirectional current, which monotonically decreases with depth.

LC is a key contributor to vertical mixing and transport of materials in the upper mixed layer of the ocean and significantly contributes to physical and geochemical processes relevant to oil spill turbulent dispersion (Lehr and Beatty 2000), ecology in the upper ocean (Dyke and Barstow 1983), and parameterization of climate models (Fox-Kemper et al. 2008). For these problems, it is important to characterize the length, velocity, and time scales of LC: (i) width of LC $L$; (ii) circulation speed of fluid within LCs $U_{d}$, or in another sense, the strength of LC; and (iii) time taken to evolve/disperse, or the characteristic time $T$. However, these important LC scales, which are dependent upon various forcing variables such as wind stress, surface wave, pycnocline depth, and so forth, are not well understood (Thorpe 2000) nor is the response joint variation of the aforementioned parameters. Moreover, their temporal evolution due to changes in direction and intensity make it difficult to estimate their dissipation time scales. This is especially true in coastal zones, the focus of this study.

As mentioned by Langmuir (1938) in his seminal paper, Langmuir cell width-half the spacing between well-defined, adjacent windrows-is difficult to measure quantitatively owing to the temporal evolution of streaks, as well as the existence of smaller streaks superimposed upon yet larger streaks. He estimated spacing to be in the range 5-25 m, which was later confirmed in lakes and ponds by Scott et al. (1969), Kenney (1977), and Thorpe and Hall (1982). Also, measurements of LC spacing varying with temporal evolution have been reported by Ichiye (1965) and Harris and Lott (1973). Although there exists a large range of scales due to variable forcing, cell spacing should be nearly constant under stable environmental conditions.

Owen (1966) reasoned that the formation of windrows is due to thermohaline convection and not due to wind forcing. Kenney (1977) hypothesized that LC spacing was independent of wind speed (based on unstratified shallow lake data). However, field observations of Zedel and Farmer (1991) and Graham and Hall (1997) pointed out that the cell width is a function of wind speed. Faller and Woodcock (1964) suggested an empirical relation, $L_{s}=4.8 s u_{w}$, where $L_{s}$ is the spacing, $s$ is seconds, and $u_{w}$ is wind speed. Katz et al. (1965) described a positive slope for the ratio $L_{s} / u_{w}$, while Maratos (1971) suggested $L_{s}=0.1 \mathrm{~m}+2.8 s u_{w}$. Such statistical correlation between LC spacing and wind speed were established from field observations, but such data were often limited by sample size and the narrow range of available wind speed. On the contrary, Scott et al. (1969) did not find a statistically significant correlation from his field observation data. Later, Faller and Caponi (1978) presented experimental results showing the relation between spacing with wavelength of surface waves by an empirical fit, given by $\lambda_{c}=$ $4.8\left[1-\exp \left(-0.5 \lambda_{w} / H\right)\right]$, where $\lambda_{c}$ is the cellular wavelength, $\lambda_{w}$ is the surface wavelength, and $H$ is the mean depth of the water column. However, they could not attribute the change in spacing to an increase in $\lambda_{w}$ (Leibovich 1983).

Langmuir (1938) suggested that cell spacing should depend upon the height of the LC $L_{h}$. Also based on his observations, he noted that the height of LC was more or less based on the thermocline depth $D_{T}$. This implies that Langmuir cells penetrated down to the thermocline depth, thereby controlling the spacing between adjacent windrows. Assaf et al. (1971) supported Langmuir's hypothesis proposing the relation between spacing and mixing layer depth $D_{m}$ to be $L_{s} / D_{m}=1.4$. Similarly, Maratos (1971) suggested $0.66 \lesssim L_{s} / D_{T} \lesssim 1.42$; Faller and Auer (1988) estimated it to be $L_{s} / H=1.5$ and Smith (1992) found it to be $L_{s} / D_{m}=2$. However, these observations can only be partially true because thermocline (or pycnocline) depth only influences $L_{h}$ if it is smaller than or comparable to $H$ (McWilliams and Sullivan 2000).

Langmuir (1938) estimated the sweeping speed (the spanwise component of the surface fluid moving toward the windrows) to be $3 \mathrm{~cm} \mathrm{~s}^{-1}$. Others (Myer 1969; Sutcliffe et al. 1963; Harris and Lott 1973; Filatov et al. 1981) measured the downwelling speed below the windrows and found it to be around $3 \mathrm{~cm} \mathrm{~s}^{-1}$, similar to the sweeping speed. In fact, these sweeping and downwelling speeds correspond to the circulation speed of LC, or, in other words, its characteristic velocity scale. Filatov et al. (1981) presented experimental results suggesting that downwelling speed and wind speed were correlated. This was in agreement with the results of Sutcliffe et al. (1963) and Harris and Lott (1973), which depicted downwelling speed increasing linearly with wind speed; however, this conclusion was made from a fit to a dataset with large scatter. Later, however, Weller and Price (1988) offered a similar conclusion from field observations, finding that circulation speed scales linearly with wind speed.

We can see in the literature that efforts to understand the scales of LC were mostly based on field observations in the real environment. Such uncontrolled studies are subjected to concerns about confounding variables because various environmental forcing conditions constantly change over time. Therefore, the purpose of this paper is to numerically investigate the factors affecting these LC scales (in coastal environments), which can be performed under controlled environmental conditions, where individual parameters can be systematically varied to assess scaling dependencies. In this study, we 
consider steady conditions with constant forcing and focus just on the length and velocity scales. However, it is acknowledged that length, velocity, and time scales are interrelated, and, therefore, deeper understanding of length and velocity scales of LC constitutes a basis for later assessments on LC time scales.

In this paper we focus our attention on understanding the "cause and effect" dynamics of environmental forcing with the length and velocity scales of LC in coastal regions. Coastal LC is a complex phenomenon, owing to highly variable wind and wave settings (Churchill et al. 2006). Gargett et al. (2004) first reported the existence of fulldepth coastal LC from their field observations at the shallow (15-m water column depth) shelf coastal region of New Jersey under strong wind and wave forcing using a five-beam acoustic Doppler current profiler with 40-cm spatial resolution. They named this LC realization "Langmuir supercells" (LSC), which fill the water column depth and couple the interactions at the surface and bottom boundary layer to enhance sediment erosion and resuspension. Later, details of the field observations (Gargett and Wells 2007) were complemented by large-eddy simulations (LES) of full-depth LC by solving the grid-filtered Craik-Leibovich equations (Tejada-Martínez and Grosch 2007; Tejada-Martínez et al. 2009), showing good agreement with the recorded observations.

Recently, Kukulka et al. $(2011,2012)$ performed LES simulations of the coastal ocean (16-m water column depth), consistent with the Coupled Boundary Layers Air-Sea Transfer (CBLAST) observations conducted on the New England shelf. Kukulka et al. (2011) showed the effect of crosswind tidal current on the structure of LC and proposed mechanisms to explain the merging and distortion of LC. Kukulka et al. (2012) studied the energetics and nonlocal transport due to LC at middepths in coastal zones. The previous simulations did not account for the effect of breaking waves. Motivated by field observations of Gerbi et al. (2009), and in order to investigate the energetics of surface boundary layer turbulence (upper $3 \mathrm{~m}$ ) due to breaking waves, Li et al. (2013) extended the LES simulations of Kukulka et al. (2012) by including the effects of wave breaking. Li et al. (2013) also studied the effect of LC and wave breaking on the surface boundary layer. They concluded that wave breaking is a dominant factor in generating surface boundary layer turbulence while LC is responsible at middepth mixing.

Gargett and Grosch (2014) attempted to further characterize LC under more complex environmental condition by adding surface cooling. They introduced new scaling (time scale characterizing growth of LC and surface friction velocity) to define two nondimensional numbers: the Langmuir number La and Rayleigh number Ra continuum. Then, they studied in detail the characteristics of situations dominated by full depth LC or full depth convection within the $\log (\mathrm{La})-\log (\mathrm{Ra})$ plane. These studies focused on either pure LC or pure convection; however, the observations made at the western shoal in the Chesapeake Bay (14-m water depth) by Scully et al. (2015) showed convection enhanced vertical mixing due to dominant LC. Later, Gargett et al. (2014) listed the set of necessary environmental conditions for the generation of Langmuir supercell events, thus describing the conditions governing the life of a LSC.

It has been shown that LC is a dominant mechanism in the study of the upper-mixed layer ocean (D'Asaro 2014). Belcher et al. (2012) pointed out that the preexisting parameterization of mixing/dissipation in global circulation models is substantially erroneous and can be corrected by including the effects of LC. Given its great importance, inclusion of Langmuir turbulence in parameterization of vertical mixing/turbulent vertical scalar fluxes has been attempted in open-ocean scenarios (D'Alessio et al. 1998; Kantha and Clayson 2004; Harcourt 2013; McWilliams and Sullivan 2000; Smyth et al. 2002) and in coastal settings (Sinha et al. 2015). In this context, LC length scale sensitivity to meteorological conditions can be an important factor to define more accurate parameterization at coastal zones. In this study we address precisely this issue.

We also aim to understand the role of a downwind pressure gradient on shallow water LC. This is another important aspect of LC studies, as it can play a vital role in the horizontal transport of materials and the transfer of momentum, heat, and mass across the air-sea interface. Most LES studies of coastal LC have considered exclusion of pressure gradient forcing (Tejada-Martínez and Grosch 2007; Tejada-Martínez et al. 2013; Sinha et al. 2015), assuming that the weak tidal velocity $\left(<50-60 \mathrm{~cm} \mathrm{~s}^{-1}\right)$ does not significantly change the characteristics of LC (Gargett et al. 2004; Gargett and Wells 2007). Also, Gerbi et al. (2009) concluded from their field observations that weak tidal currents interact only near the bottom boundary layer and do not impact the upper mixed layer, and this was supported by LES of LC by Li et al. (2008, 2010, 2013). Martinat et al. (2011) also attempted to understand the interaction of turbulence generated by wind-driven flows and pressure-driven flows with wave forcing aligned in the normal and tangential direction. Also, Kukulka et al. (2011) attempted to understand the effect of crosswind tidal current on coastal LC and proposed two mechanisms explaining the distortion of LC due to imposed crosswind pressure gradient. In our study, we also consider a downwind pressure gradient force to investigate its effect on the length scales and structure of LC.

The paper is organized as follows. In section 2, a brief description of the governing equations and numerical procedure is presented. Then, in section 3, we will 
TABLE 1. List of numerical experiments used to assess the length scales of coastal LC. In the table, bold values indicate those simulation parameters that are varied while others are fixed. Also, $N^{*}=N_{x}=N_{y}=N_{z}$ and $L^{\dagger}=\left(L_{x}, L_{y}, L_{z}\right)$.

\begin{tabular}{|c|c|c|c|c|c|c|c|c|}
\hline Case & $U_{s} / u_{*}$ & $u_{w} / u_{*}$ & $k H$ & $\Pi$ & Coriolis & Buoyancy & Grid $\left(N^{*}\right)$ & Domain $\left(L^{\dagger}\right)$ \\
\hline$O_{\mathrm{CB}}$ & 15.74 & 1.41 & 9.45 & 0 & Yes & Yes & 192 & $2,2,1$ \\
\hline$O_{\mathrm{NCB}}$ & 15.74 & 1.41 & 9.45 & 0 & No & No & 192 & $2,2,1$ \\
\hline$C_{U_{s}(1), u_{w}(1), k H(1), N(1), L(1)}$ & 4.34 & 1 & 1.2 & 0 & No & No & 192 & $\mathbf{2} \pi, \mathbf{2} \pi, \mathbf{1}$ \\
\hline$C_{U_{s}(2)}$ & 16.95 & 1 & 1.2 & 0 & No & No & 192 & $2 \pi, 2 \pi, 1$ \\
\hline$C_{U_{s}(3)}$ & 2.82 & 1 & 1.2 & 0 & No & No & 192 & $2 \pi, 2 \pi, 1$ \\
\hline$C_{U_{s}(4)}$ & 1.41 & 1 & 1.2 & 0 & No & No & 192 & $2 \pi, 2 \pi, 1$ \\
\hline$C_{u_{w}(2)}$ & 4.34 & 0.65 & 1.2 & 0 & No & No & 192 & $2 \pi, 2 \pi, 1$ \\
\hline$C_{u_{w}(3)}$ & 4.34 & 0.5 & 1.2 & 0 & No & No & 192 & $2 \pi, 2 \pi, 1$ \\
\hline$C_{u_{w}(4)}$ & 4.34 & 0.4 & 1.2 & 0 & No & No & 192 & $2 \pi, 2 \pi, 1$ \\
\hline$C_{k H(2)}$ & 4.34 & 1 & 10 & 0 & No & No & 192 & $2 \pi, 2 \pi, 1$ \\
\hline$C_{N(2)}$ & 4.34 & 1 & 1.2 & 0 & No & No & 128 & $2 \pi, 2 \pi, 1$ \\
\hline$C_{L(2)}$ & 4.34 & 1 & 1.2 & 0 & No & No & 256 & $8 \pi / 3,8 \pi / 3,1$ \\
\hline$C \prod_{U_{s}(1), u_{w}(1), k H(1)}$ & 15 & 1.41 & 1.2 & 1 & No & No & 192 & $2 \pi, 2 \pi, 1$ \\
\hline$C \Pi_{U_{s}(2)}$ & 30 & 1.41 & 1.2 & 1 & No & No & 192 & $2 \pi, 2 \pi, 1$ \\
\hline$C \Pi_{U_{s}(3)}$ & 2 & 1.41 & 1.2 & 1 & No & No & 192 & $2 \pi, 2 \pi, 1$ \\
\hline$C \Pi_{U_{s}(4)}$ & 4 & 1.41 & 1.2 & 1 & No & No & 192 & $2 \pi, 2 \pi, 1$ \\
\hline$C \Pi_{U_{s}(5)}$ & 7 & 1.41 & 1.2 & 1 & No & No & 192 & $2 \pi, 2 \pi, 1$ \\
\hline$C \Pi_{u_{w}(2)}$ & 15 & 0.71 & 1.2 & 1 & No & No & 192 & $2 \pi, 2 \pi, 1$ \\
\hline$C \Pi_{u_{w}(3)}$ & 15 & 1 & 1.2 & 1 & No & No & 192 & $2 \pi, 2 \pi, 1$ \\
\hline$C \prod_{u_{w}(4)}$ & 15 & 2 & 1.2 & 1 & No & No & 192 & $2 \pi, 2 \pi, 1$ \\
\hline$C \Pi_{k H(2)}$ & 15 & 1.41 & 10 & 1 & No & No & 192 & $2 \pi, 2 \pi, 1$ \\
\hline
\end{tabular}

highlight the validation of Langmuir turbulence both in open-ocean settings (section 3a) and in coastal-ocean settings (section $3 \mathrm{~b}$ ), to demonstrate the efficacy of the numerical procedure. We report results in section 4 , wherein we outline the influence of environmental parameters on LC length scales. This is followed by comments on the structural difference in open and coastal Langmuir turbulence in section 5. Final remarks are reported in the conclusion. Appendixes also supplement the paper: appendix A discusses the effect of resolution and domain size on the simulations, appendix B investigates the influence of pressure gradient force on $\mathrm{LC}$ length scales, and appendix C lists supplementary simulations to support Table 1.

\section{Governing equations and cases}

The LES code solves the low-pass (grid) filtered, nondimensional Craik-Leibovich equations for transport of momentum $\tilde{\mathbf{u}}(\mathbf{x}, t)$, as follows:

$$
\begin{aligned}
\frac{\partial \tilde{\mathbf{u}}}{\partial t}+\frac{1}{2} \nabla(\tilde{\mathbf{u}} \cdot \tilde{\mathbf{u}})-\tilde{\mathbf{u}} \times \tilde{\boldsymbol{\omega}}= & -\nabla \tilde{p}-\nabla \cdot \boldsymbol{\tau}+\mathbf{e}_{x} \Pi \\
& -\frac{1}{\operatorname{Ro}} \mathbf{F}+\operatorname{Ri}\left(\tilde{\theta}-\theta_{b}\right) \mathbf{e}_{z} \\
& +\frac{1}{\mathrm{La}_{t}^{2}}\left(\mathbf{u}_{s} \times \tilde{\boldsymbol{\omega}}\right),
\end{aligned}
$$

where $\tilde{\boldsymbol{\omega}}=\nabla \times \tilde{\mathbf{u}}$ is vorticity; $\nabla \tilde{p}$ is pressure correction; $\boldsymbol{\tau}=\overline{\mathbf{u}}^{\prime} \cdot \mathbf{u}^{\prime}-\tilde{\mathbf{u}}^{\prime} \cdot \tilde{\mathbf{u}}^{\prime}$ (prime denotes fluctuations) is the subgrid-scale (SGS) stress tensor (Germano et al. 1991;
Mason 1994; Meneveau and Katz 2000; Bou-Zeid et al. 2005); $\mathbf{e}_{x}$ and $\mathbf{e}_{z}$ are unit vectors in the streamwise and vertical directions, respectively; $\Pi=\left(d P_{0} / d x\right) H / \rho=1$ is a pressure gradient forcing, where $H$ is the column depth and $u_{\tau}$ is a shear velocity associated with ambient pressure gradient forcing; Ro is the Rossby number and $\mathbf{F}$ is the Stokes-modified Coriolis force (Tejada-Martínez et al. 2009); $\mathrm{Ri}$ is the Richardson number; $\tilde{\theta}$ is the filtered temperature and $\theta_{b}$ is the bulk temperature obtained after averaging over the horizontal direction $x, y$ (TejadaMartínez et al. 2009); and $\mathbf{u}_{s} \times \tilde{\boldsymbol{\omega}}$ is a vortex force related to tilting of vertical vorticity via Stokes drift. For the case simulated here,

$$
\begin{aligned}
\mathbf{u}_{s} \times \tilde{\boldsymbol{\omega}}= & \epsilon_{i j k} u_{s, j} \tilde{\omega}_{k}=\left(u_{s, 2} \tilde{\omega}_{3}-u_{s, 3} \tilde{\omega}_{2}\right) \hat{\mathbf{i}} \\
& +\left(u_{s, 3} \tilde{\omega}_{1}-u_{s, 1} \tilde{\boldsymbol{\omega}}_{3}\right) \hat{\mathbf{j}}+\left(u_{s, 1} \tilde{\omega}_{2}-u_{s, 2} \tilde{\omega}_{1}\right) \hat{\mathbf{k}} \\
= & 0 \hat{\mathbf{i}}-u_{s, 1} \tilde{\omega}_{3} \hat{\mathbf{j}}+u_{s, 1} \tilde{\omega}_{2} \hat{\mathbf{k}},
\end{aligned}
$$

where the subscript comma serves only to separate notation, not to indicate derivatives, and $\epsilon_{i j k}$ denotes the Levi-Civita symbol. This LES code is an extension of the Johns Hopkins University (JHU) LES code with the additional vortex forcing. The code is well tested and has been applied to diverse turbulent boundary layer studies [development of SGS parameterization schemes (Bou-Zeid et al. 2005), modeling fully developed flows over complex topography (Anderson and Meneveau 2011; Anderson and Chamecki 2014), and modeling flow over vegetative canopies (Chester et al. 2007; Graham and 
Meneveau 2012)]. Owing to the high Reynolds number conditions, viscous stresses are neglected. Incompressibility is attained via computation of the divergence of Eq. (1) and solution of the resultant pressure Poisson equation for a pressure correction. Periodic boundary conditions are applied in the horizontal directions by virtue of spectral discretization in the horizontal directions. Boundary conditions at the top of the domain boundary are imposed with a constant aerodynamic surface stress aligned with the $x$ direction (Fig. 1), which captures the imposed aerodynamic loading due to the aloft atmospheric surface layer and the nonpenetration condition for vertical velocity, $\left.\tilde{w}\right|_{z / H=1}=0$. Centered second-order finite differencing is used to evaluate vertical gradients, and the second-order Adams-Bashforth scheme has been used for time integration. The nonlinear advective term is dealiased with the $3 / 2$ rule (Orszag 1970).

The deviatoric component of SGS stress is parameterized using an eddy-viscosity model:

$$
\boldsymbol{\tau}-\frac{1}{3} \boldsymbol{\delta} \operatorname{Tr}(\boldsymbol{\tau})=-2 \nu_{t} \tilde{\mathbf{S}}
$$

where $\nu_{t}=\left(C_{s} \Delta\right)^{2}|\tilde{S}|$ is the eddy viscosity; $C_{s}$ is the Smagorinsky coefficient, evaluated here using the Lagrangian scale-dependent dynamic SGS model of Bou-Zeid et al. (2005); $\Delta$ is the filter size; $\tilde{\mathbf{S}}$ is the resolved strain-rate tensor; and $|\tilde{S}|=(2 \tilde{\mathbf{S}}: \tilde{\mathbf{S}})^{1 / 2}$ is the magnitude of the resolved strain-rate tensor.

For the bottom wall boundary conditions, in practice there are two approaches to model near-wall Langmuir turbulence: (i) resolving the near-wall turbulence (Tejada-Martínez and Grosch 2007) or (ii) using a walllayer model (Piomelli and Balaras 2002). The first approach can resolve the small-scale turbulence near a solid boundary but demands higher computational cost with increasing Reynolds number. For this reason, Tejada-Martínez and Grosch (2007) selected a lower Reynolds number, while fully resolving the near-wall turbulence. They showed that their LES results matched up well with field observations. The second approach is based on the logarithmic law of the wall and allows simulation of high Reynolds number flows. TejadaMartínez et al. (2012) have shown that in this case, LC disrupts the logarithmic layer near the bottom wall, and therefore it is inappropriate to presume the existence of logarithmic conditions. However, their study also indicates that the disruption is unlikely to be significant as $\mathrm{La}_{t}$ and $\Lambda$ (the wavelength of the surface waves) approach 0.7 and $1.33 H$, respectively. In the present work, we nonetheless adopt the logarithmic equilibrium wall model, fully cognizant of the deviation from logarithmic conditions beneath Langmuir turbulence. This is still

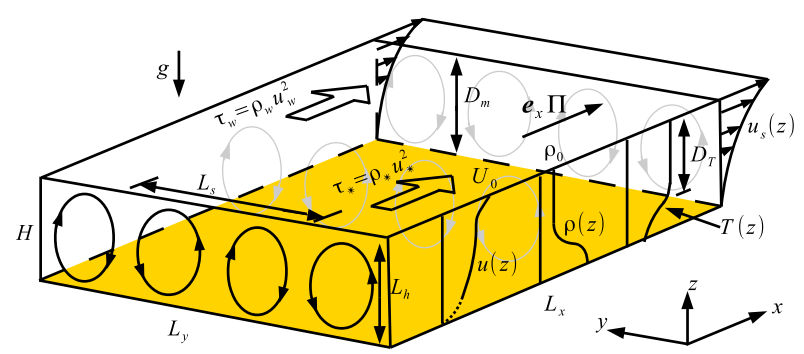

FIG. 1. Sketch of computational domain showing spatial extent in the streamwise $L_{x}$, spanwise $L_{y}$, and column depth $H$ directions. The sketch includes annotations of $x$-aligned shear imposed by the surface wind $\tau_{w}$ and associated with bathymetric drag in coastal zones $\tau_{*}$. Langmuir circulations (black and gray) and seafloor in yellow. The sketch shows the Stokes profile, along with spacing and depth of Langmuir cells $L_{s}$ and $L_{h}$, respectively, temperature and density profiles $T(z)$ and $\rho(z)$, respectively, and pycnocline depth $D_{T}$ (shown also is the surface density $\rho_{0}$ and acceleration due to gravity $g$ ). Shown also is the streamwise velocity profile $u(z)$ and "outer" velocity $U_{0}$, where the dashed profile at the bottom indicates differences in this profile between the open and coastal ocean. In this sketch, the column and mixed layer depth $D_{m}$ and $H$ are presumed equivalent, although this need not be the case.

reasonable, though, since some model is required to impose surface stress, and the logarithmic model has been shown to exhibit great versatility in such applications (Willingham et al. 2013). In this case, the logarithmic model is presented in an alternative form via introduction of a momentum transfer function:

$$
\begin{aligned}
\tau_{i z}^{w}(x, y, t) & =-\left[\frac{\kappa}{\ln \left(z / z_{0}\right)}\right]^{2} U(x, y, z, t)^{2} \hat{\mathbf{i}}_{u} \\
& =C_{M} U(x, y, z, t)^{2} \hat{\mathbf{i}}_{u},
\end{aligned}
$$

where $i=x, y, \hat{\mathbf{i}}_{u}=\tilde{u}_{i} / U$ is a local unit vector on velocity, $\kappa$ is the von Kármán constant, $z_{0} / H=10^{-3}$ is the local roughness length, and $U=\left(\tilde{u}^{2}+\tilde{v}^{2}\right)^{1 / 2}$ is the magnitude of resolved LES velocity at height $z$.

Persistent orbital dynamics of wind-driven surface waves and associated tilting of vertical vorticity are responsible for sustenance of Langmuir circulations. These effects can be characterized via the Langmuir number $\mathrm{La}_{t}$ and parameterized via the one-dimensional Stokes drift (Phillips 1966). Nondimensional Stokes drift velocity is defined as follows:

$$
U_{s}^{1}=\frac{\cosh \left[2 k\left(z_{1}+H\right)\right]}{2 \sinh ^{2}(k H)} ; \quad U_{s}^{2}=U_{s}^{3}=0,
$$

where $z_{1}=0$ is the average surface height, $H$ is the water depth, and $k$ is the dominant wavenumber of the surface gravity wave. Here, we only consider the dominant wave, which is a representative monochromatic wave from the broadband spectrum of the wave field observed 
in nature. More specifically, in this work [motivated from the field observations of LSC by Gargett and Wells (2007) and complementary LES study by Tejada-Martínez and Grosch (2007)] the dominant wave associates with the high-frequency surface wave peak of the spectrum of vertical velocity [observed in Fig. 3 of Gargett and Wells (2007)]. The turbulent Langmuir number is defined as follows (McWilliams et al. 1997):

$$
\mathrm{La}_{t}=\left(\frac{u_{w}}{U_{s}}\right)^{1 / 2}=\left(\frac{u_{w}}{u_{*}} \frac{u_{*}}{U_{s}}\right)^{1 / 2},
$$

where $u_{w}$ and $u_{*}$ correspond to shear velocities due to top surface wind stress and bottom wall stress, respectively. Here, Stokes drift surface velocity is $U_{s}=$ $\sigma_{w} k a^{2} \operatorname{coth} k H$, where $\sigma_{w}=(g k \tanh k H)^{1 / 2}$ is the dominant frequency, $k=2 \pi / \lambda$ is the dominant wavenumber, and $a$ is the amplitude of surface gravity waves [see Gargett and Wells (2007) for calculation of these wave characteristics from field measurements].

Moreover, we emphasize here that our LES simulations with coastal-ocean settings do not consider rotational effects [Coriolis accelerations omitted from Eq. (1)]. In the literature, most LES-based studies omit Coriolis forcing in their model (Tejada-Martínez and Grosch 2007; Martinat et al. 2011; Akan et al. 2013; Kukulka et al. 2012), although Li et al. (2013) did not, assuming that its effect is insignificant on coastal LC. It was believed to be valid based on good agreement of LES results with observational data. More recently, Grosch and Gargett (2016) demonstrated through theoretical analysis that LC generated with rotational effects and lateral boundary (defined based on field observations) were similar in nature to the case without rotational effects and lateral boundary, thus validating the use of nonrotational LES of LC.

\section{Cases}

Table 1 summarizes different configurations used to investigate the factors influencing LC height, width, and strength. For these cases, certain parameters are carefully varied while others are fixed, which reveal the system sensitivity to these parameters. In the table, first there are two open-ocean cases indicated as $O_{\mathrm{CB}}$ and $O_{\mathrm{NCB}}$ to represent the flow with and without Coriolis and buoyancy forcing, respectively. Then, a series of coastal-ocean cases are listed, uniquely identified by the given nomenclature $C_{X(Y)}$. Here, $C$ denotes coastal ocean, $X$ denotes the forcing parameter whose magnitude is allowed to vary while other parameters remain unchanged, and $Y$ denotes numbering of that varying forcing parameter. For example, $C_{u_{w}(2)}$ points out to a coastal-ocean case with a second variation of the magnitude of the wind stress forcing parameter. Here, $U_{s}$ represents Stokes drift surface velocity, $u_{w}$ represents surface wind stress, $k H$ represents wavenumber, $N$ represents grid resolution, and $L$ represents domain size. Also, a series of cases with axial pressure gradient forcing is included. It is designated as $C \Pi_{X(Y)}$, where $\Pi$ denotes active axial pressure gradient forcing and all other symbols carry the same meaning as above. Also, to note in the table are two base cases $C_{U_{s}(1), u_{w}(1), k H(1), G(1), L(1)}$ and $C \Pi_{U_{s}(1), u_{w}(1), k H(1)}$, which are the starting points to each parametric study.

\section{Validation}

\section{a. Open-ocean settings}

For validation purposes, we want to compare our LES results with the benchmark dataset of McWilliams et al. (1997), as well as the dataset of Yang et al. (2015). We repeat the simulations by matching our LES simulations with their simulation setup. Constant wind stress $\left(0.037 \mathrm{~N} \mathrm{~m}^{-2}\right)$, corresponding to a wind speed of $5 \mathrm{~m} \mathrm{~s}^{-1}$ at $10-\mathrm{m}$ height, is applied on the top boundary and aligned with the wave field in the streamwise direction. This results in an ocean surface friction velocity of $u_{w}=$ $6.1 \times 10^{-3} \mathrm{~m} \mathrm{~s}^{-1}$. Coaligned surface waves have wavelength $\Lambda=60 \mathrm{~m}$ and amplitude $a=0.8 \mathrm{~m}$ indicating wavenumber $k=0.105 \mathrm{~m}^{-1}$ and Stokes surface velocity $U_{s}=0.068 \mathrm{~m} \mathrm{~s}^{-1}$. This gives a turbulent Langmuir number of $\mathrm{La}_{t}=\sqrt{u_{w} / U_{s}}=0.2997$. The computational domain is a "half-channel flow" with imposed surface stress at the centerline, with a rectangular domain of nondimensional horizontal size, $L_{x}=L_{y}=2$, and depth $H=1$ (or, $180 \mathrm{~m} \times 180 \mathrm{~m} \times 90 \mathrm{~m}$ ). The computational grid size is $192 \times 192 \times 192$.

In this test, we also have Coriolis and buoyancy forcing activated. Corresponding to a latitude of $45^{\circ} \mathrm{N}$, the Coriolis frequency $f_{c}=2 \Omega \sin \left(45^{\circ}\right)$, is calculated to be $10^{-4} \mathrm{~s}^{-1}$ (where $\Omega$ is the rotation rate of Earth). The thermocline depth is defined at $z_{i}=-33 \mathrm{~m}$ from the ocean surface, which is the mixing layer depth for a constant-density profile. Below the thermocline, the density profile is stably stratified, which hinders vertical mixing capacity of Langmuir turbulence and attenuates vertical fluctuations associated with LC. For simplicity, in our LES simulations, the buoyancy force is imposed with a nonupdating density profile - the density profile corresponds to a flow field that is well mixed above $z / z_{i}=-1$ and stably stratified below, agreeing with the description in McWilliams et al. (1997)—-thereby eliminating the need to model active scalar transport. This approach is implemented for simplicity, at the expense of higher accuracy, unlike the LES simulations by McWilliams et al. (1997). However, we consider this 

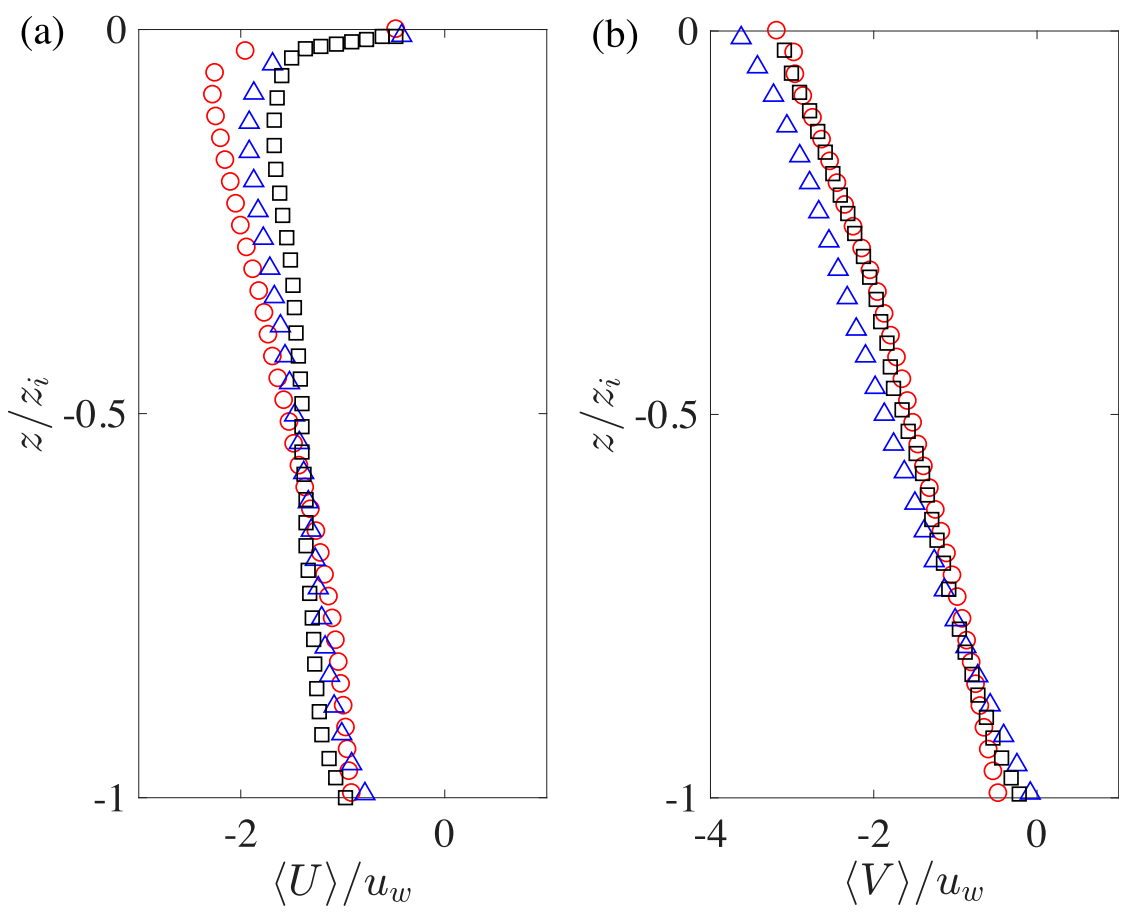

FIG. 2. Time- and horizontally averaged vertical profiles normalized by the aerodynamic surface stress $u_{w}$ : mean (a) streamwise and (b) spanwise velocity. Black squares indicate the dataset of McWilliams et al. (1997), blue triangles indicate the dataset of Yang et al. (2015), and red circles are our LES results.

approach to reproduce similar characteristics of openocean Langmuir turbulence with minimal discrepancies.

Figures 2-4 compare the first- and second-order turbulence quantities with the dataset of McWilliams et al. (1997) and Yang et al. (2015). Following the addition of the vortex force with Coriolis force and buoyancy force, the system responds by producing Langmuir cells. This induces inertial oscillations that have been eliminated (from the statistics) by time averaging over $7.1 T_{1}$, where $T_{1}$ is the nondimensional inertial time period. It has been shown by Zikanov et al. (2003) that at least averaging over $5 T_{1}$ is necessary to attain stationary flow statistics. Also, the condition, given by $\int\langle\tilde{u}\rangle_{T} d z+\int\left(U_{s}^{1} / \mathrm{La}_{t}^{2}\right) d z=0$ (TejadaMartínez et al. 2009), is satisfied with the two terms within $2.9 \%$ of each other in magnitude. Figure 2 a shows a time and horizontally averaged vertical profile of mean streamwise velocity. As explained by McWilliams et al. (1997), the maximum difference between Langmuir turbulence and shear turbulence is seen in the vertical profile of mean streamwise velocity. It is quite uniformly distributed with depth, representing the homogenization process associated with Langmuir turbulence and thus elevated vertical mixing and transport. Also the negative values of $\langle U\rangle$ explain that Langmuir turbulence depletes momentum from the streamwise direction in order to sustain spanwise and vertical momentum.
Similar characteristics of $\langle U\rangle$ in the coastal-ocean case have been reported in section $3 b$, differing only at the bottom where shear associated with the bottom-boundary layer dominates. In Fig. 2a, our LES results show similar characteristics of the flow as explained above. Figure $2 b$ shows the vertical profile of mean spanwise velocity. Above the thermocline depth, $\langle V\rangle$ deviates significantly from zero as a result of the Coriolis effect, which is not the case otherwise. This flow component also explains the existence of spanwise convergence and divergence motions, due to counterrotating vortex pairs.

Figure 3 shows the vertical profiles of mean vertical momentum fluxes, which is more relevant to vertical transport capacity in the open-ocean settings. It is clearly evident in Fig. 3a that $\left\langle\tilde{u}^{\prime} \tilde{w}^{\prime}\right\rangle$ has near zero magnitude and $\left\langle\tilde{\boldsymbol{v}}^{\prime} \tilde{w}^{\prime}\right\rangle$ has very small magnitude below the thermocline depth (note that the Reynolds stresses are assembled via addition of the turbulent stresses developed by the resolved and subgrid-scale flow). This means that below the thermocline, there is no contribution of these momentum fluxes to the momentum balance. Anisotropy of the Langmuir turbulence can easily be understood from the Reynolds normal stress profiles in Fig. 4. As a result of enhanced upwelling and downwelling motion above the thermocline depth, $\left\langle\tilde{w}^{\prime} \tilde{w}^{\prime}\right\rangle$ increases but $\left\langle\tilde{u}^{\prime} \tilde{u}^{\prime}\right\rangle$ decreases. This happens because 

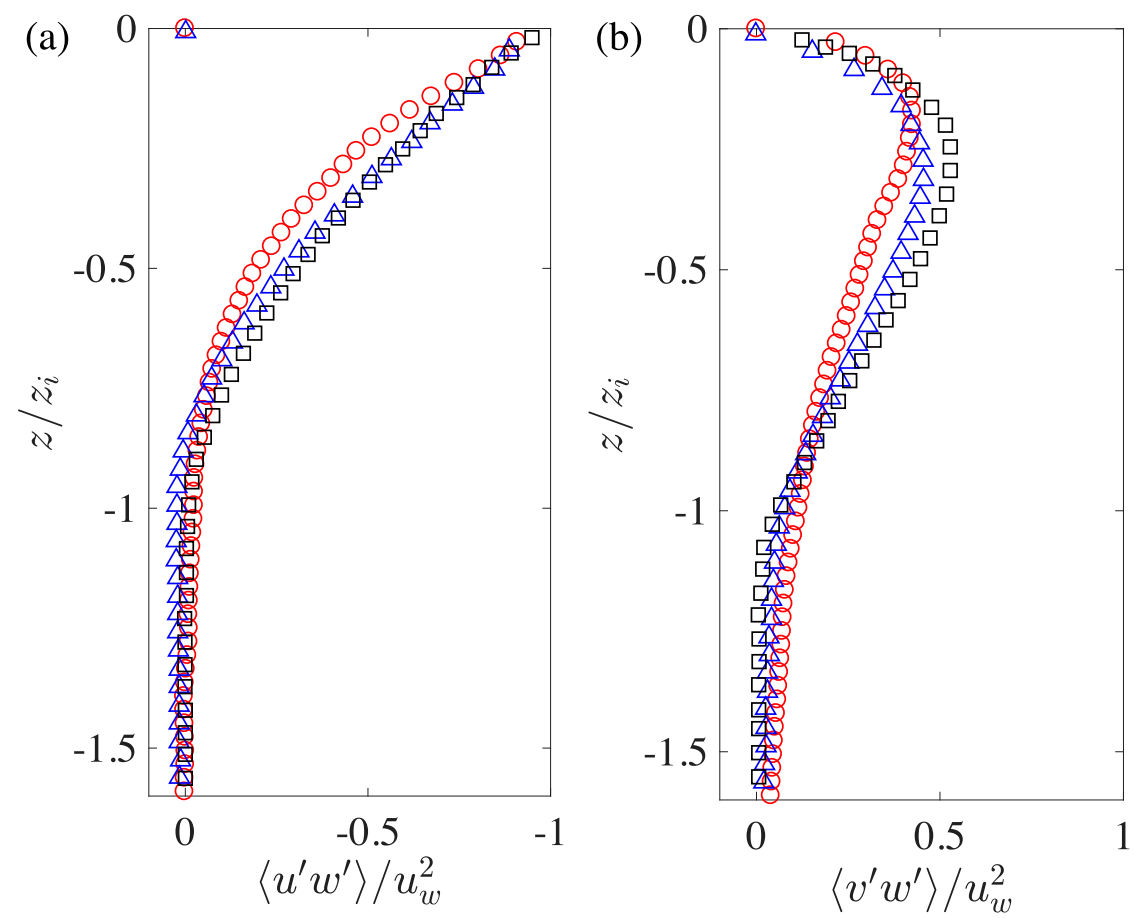

FIG. 3. Time- and horizontally averaged vertical profiles of momentum fluxes, normalized by $u_{w}^{2}$ : (a) streamwise-vertical and (b) spanwise-vertical Reynolds shear stresses. Black squares indicate the dataset of McWilliams et al. (1997), blue triangles indicate the dataset of Yang et al. (2015), and red circles are our LES results.

kinetic energy production switches from the streamwise component of shear production into the vertical component of CL vortex production. The large magnitude of $\left\langle\tilde{\boldsymbol{v}}^{\prime} \tilde{\boldsymbol{v}}^{\prime}\right\rangle$ explains the convergence and divergence zones of counterrotating Langmuir cells. The hierarchy of magnitude of Reynolds normal stress components can be listed as $\left\langle\tilde{\boldsymbol{v}}^{\prime} \tilde{\boldsymbol{v}}^{\prime}\right\rangle>\left\langle\tilde{u}^{\prime} \tilde{u}^{\prime}\right\rangle>\left\langle\tilde{w}^{\prime} \tilde{w}^{\prime}\right\rangle$. Our LES results agree well with the datasets of McWilliams et al. (1997) and Yang et al. (2015).

\section{b. Coastal-ocean settings}

Proceeding with the coastal-ocean cases, we ran LES simulations replicating the field observations at the shallow ( $H=15$-m water depth) shelf coastal region of New Jersey (record 43.025) (Gargett et al. 2004; Gargett and Wells 2007). The recorded intermediate waves had frequency $\sigma_{w}=0.82 \mathrm{rad} \mathrm{s}^{-1}$; wavenumber $k=0.08 \mathrm{rad} \mathrm{m}^{-1}$ corresponding to wavelength $\Lambda=79 \mathrm{~m}(\Lambda / H \sim 5)$ and amplitude $a=0.7 \mathrm{~m}$. Calculations show that Stokes drift surface velocity $U_{s}=\sigma_{w} k a^{2} \operatorname{coth}(k H)=0.038 \mathrm{~m} \mathrm{~s}^{-1}$ and top surface wind stress $\tau_{w}=0.079 \mathrm{~N} \mathrm{~m}^{-2}$ correspond to surface friction velocity $u_{w}=0.00889 \mathrm{~m} \mathrm{~s}^{-1}$. This results in a turbulent Langmuir number [Eq. (6)] of $\mathrm{La}_{t}=$ 0.48 and nondimensional wavenumber $k H=1.2$. The computational domain was a half-channel flow, with a rectangular domain of spatial extent of $L_{x}=L_{y}=2 \Pi$ and depth $H=1$ (or $L_{x}=94.25 \mathrm{~m}, L_{y}=94.25 \mathrm{~m}$, $H=15 \mathrm{~m}$ ) and the computational grid size $192 \times 192 \times$ 192. The wind and wave forcing is aligned in the streamwise direction (Fig. 1). For this validation case corresponding to field observation record 43.025, we do not impose a downwind pressure gradient force. The effect of the imposed pressure gradient force on length scales of LC will be detailed in appendix B. Coriolis and buoyancy forcing has been deactivated, following preceding studies by Tejada-Martínez and Grosch (2007) and Kukulka et al. (2012). The usage of nonrotational LES of LC has also been validated very recently by Grosch and Gargett (2016) through theoretical analysis. In this LES, under statistical equilibrium the top surface wind shear stress is equal to the bottom wall shear stress (Tejada-Martínez and Grosch 2007). Therefore,

$$
\begin{aligned}
& \lambda=\frac{u_{w}}{u_{*}}=\left(\frac{\tau_{w} \rho_{w}^{-1}}{\tau_{*} \rho_{*}^{-1}}\right)^{1 / 2}=1, \quad \text { or } \\
& \Psi=\left(\frac{\tau_{w}}{\tau_{*}}\right)=1
\end{aligned}
$$

where $u_{w}$ is surface friction velocity, $u_{*}$ is bottom friction velocity, $\tau_{w}$ is top-surface wind stress, and $\tau_{*}$ is 

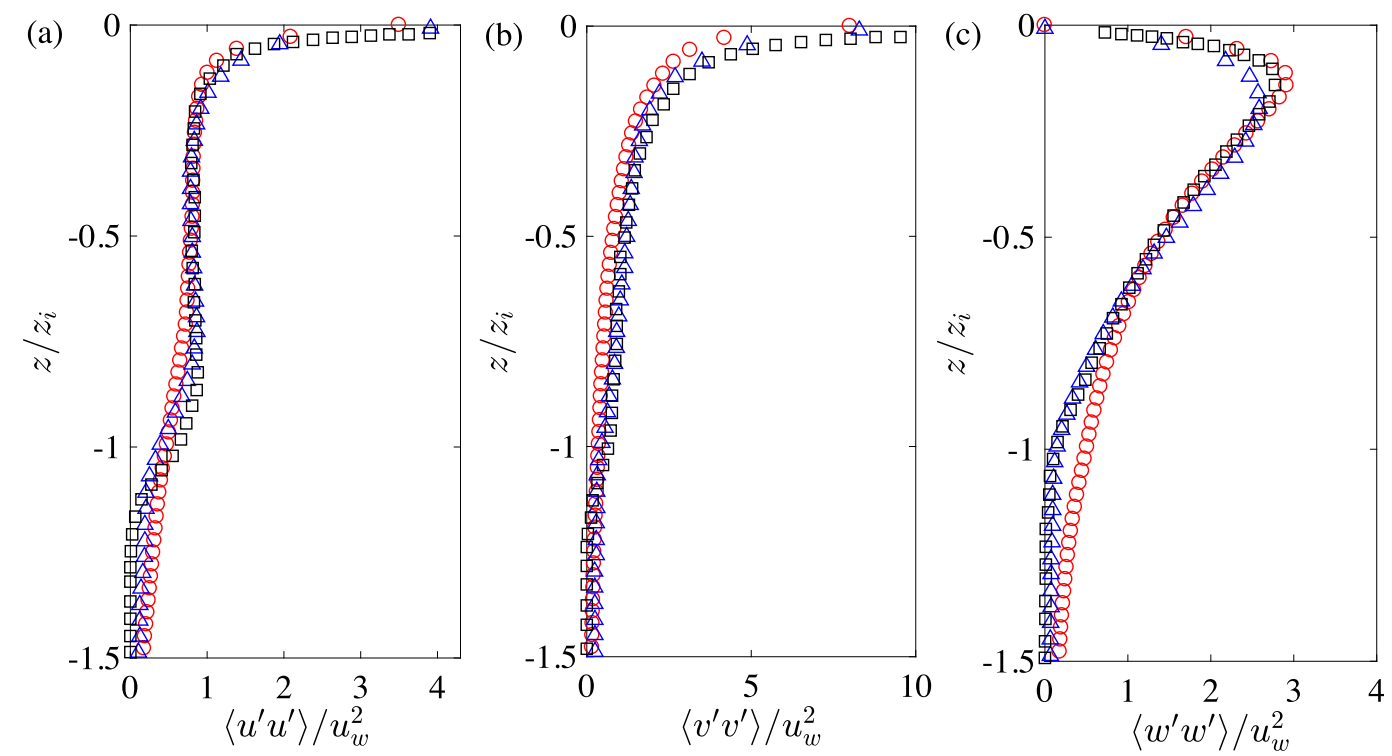

FIG. 4. Time- and horizontally averaged vertical profiles of velocity variances, normalized by $u_{w}^{2}$ : (a) streamwise, (b) spanwise, and (c) vertical Reynolds normal stresses. Black squares indicate the dataset of McWilliams et al. (1997), blue triangles indicate the dataset of Yang et al. (2015), and red circles are our LES results.

bottom-boundary layer surface stress (Martinat et al. 2011; Gargett and Grosch 2014).

Figure 5 shows contours of instantaneous streamwise, spanwise, and vertical velocity in the spanwise-vertical $(y-z)$ plane at streamwise location $x=L_{x} / 3$. The panel also includes streamwise-averaged streamwise vorticity. This is the dynamically relevant plane since it qualitatively illustrates the presence of the counterrotating Langmuir cells. The contours highlight how the momentum field varies due to the vortex forcing and imposed wind stress. Figure 5a shows the instantaneous streamwise velocity (retrieved from the LES velocity field). The contour shows the presence of roll cells with alternating momentum (from regions of momentum excess to deficit). This is more clearly shown via the contours of spanwise and vertical velocity in Figs. 5b and $5 \mathrm{c}$, respectively, which show the alternating patterns of upwelling and downwelling that are the signature of Langmuir turbulence. Also, Fig. $5 \mathrm{c}$ is consistent with field observations by Gargett et al. (2004) and Gargett and Wells (2007) that downwelling regions are narrower than upwelling regions. Finally, the contour of streamwise vorticity (Fig. 5d) comprehensively shows how LC occupies the column. We emphasize, once more, that these cells would vanish in the absence of vortex forcing, in which case the flow would tend toward a canonical turbulent channel flow.

Figure 6a shows $\langle U\rangle(z)$, a vertical profile of streamwise velocity. Brackets \langle\rangle represents time and horizontal averages of the quantity. The profile exhibits a zero slope at middepth and negative slope in the upper part of the water column, indicating the effect of the imposed wind stress $\tau_{U}^{w}$. The imposed vortex forcing $\mathbf{f}_{L}$ induces Langmuir cells that extract momentum from the streamwise direction. Therefore, $\langle U\rangle$ is almost $z$ invariant at the middepth of the water column. Figures $6 \mathrm{~b}$ and $6 \mathrm{c}$ show vertical profiles of the Reynolds (turbulent) normal and shear stresses. Here, the profiles demonstrate how mixing varies over the water column, owing to the combined effects of the vortex force and imposed wind stress. The profile for normal streamwise stress $\left\langle\tilde{u}^{\prime} \tilde{u}^{\prime}\right\rangle$ is primarily attributed to the bottom wall stress and surface wind stress, and as such the elevated stresses in the bottom boundary layer and upper mixed layer are apparent. The spanwise and vertical normal stresses are, on the other hand, a manifestation of the imposed vortex forcing and Langmuir turbulence. The spanwise normal stresses are almost equivalent to the normal streamwise stresses in the upper mixed layer (due to shear associated with the counterrotating Langmuir cells), and the vertical normal stresses-which would otherwise be a small fraction of the other components of normal stresses in the absence of vortex forcing-actually are equivalent to the streamwise and spanwise normal stresses in the midregion. This distribution is a consequence of the vortex forcing-induced Langmuir turbulence and highlights the much greater vertical transport potential. The above elaboration of the characteristics 

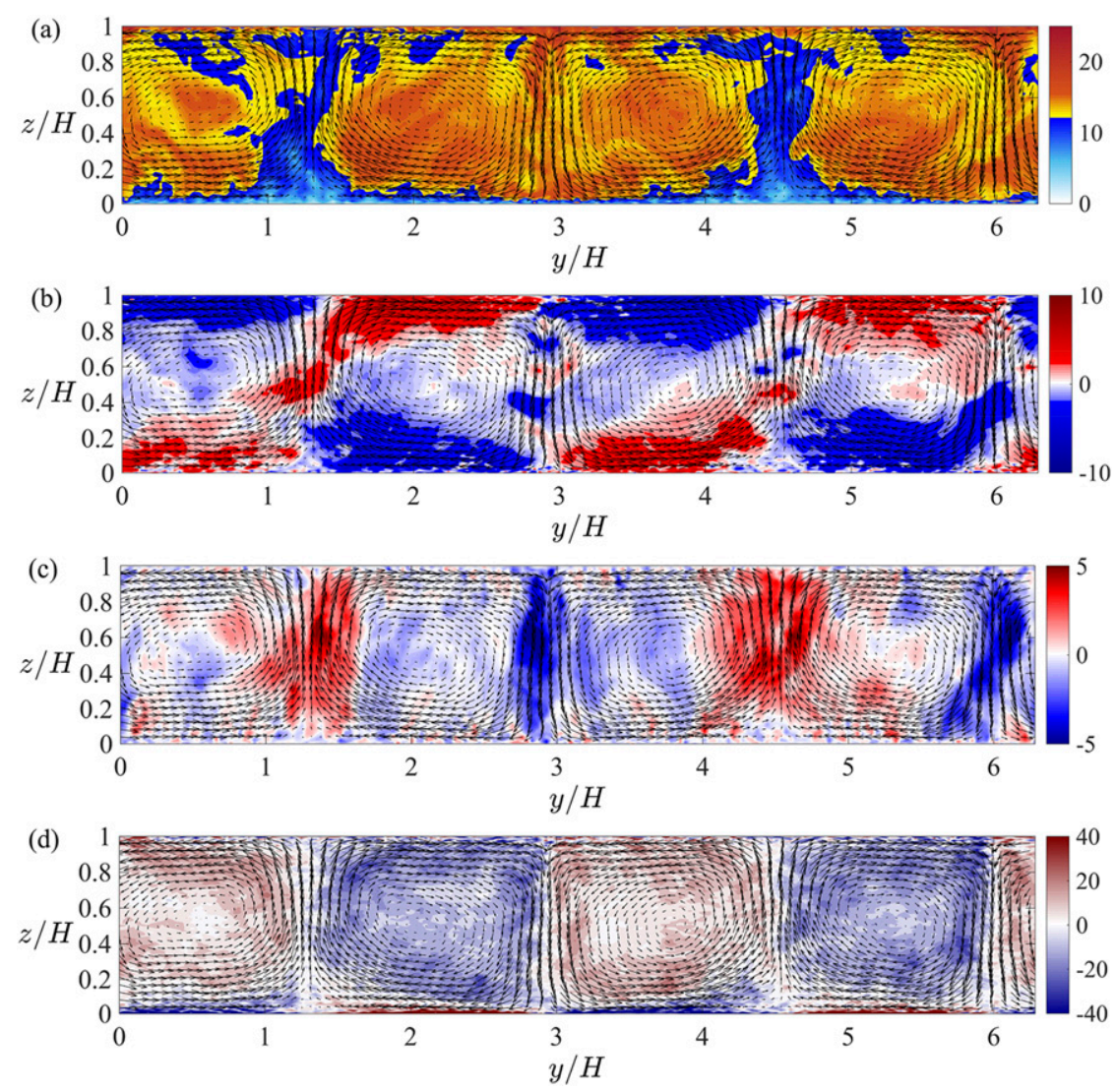

FIG. 5. Instantaneous velocity contours at $L_{x} / 3$ plane of the flow referring to case $C_{U_{s}(1), u_{w}(1), k H(1), N(1), L(1)}$ (a) instantaneous streamwise velocity $\tilde{u}$, (b) instantaneous spanwise velocity $\tilde{v}$, (c) instantaneous vertical velocity $\tilde{w}$, and (d) streamwise vorticity averaged along streamwise direction $\tilde{\omega}_{x}$. Note that the vorticity is calculated using the gradients of instantaneous velocity and not the gradients of mean velocity. Here $z / H=0$ denotes the bottom of the water column, and $z / H=1$ denotes the water surface. Horizontal axis denotes crosswind direction.

of coastal Langmuir turbulence very well match the findings of antecedent studies (cf. Tejada-Martínez and Grosch 2007; Kukulka et al. 2012; Tejada-Martínez et al. 2013; Akan et al. 2013; Sinha et al. 2015; Walker et al. 2016).

\section{Parameters controlling scales of LC}

The majority of previous research on Langmuir turbulence has focused on deep water scenarios (McWilliams et al. 1997; Yang et al. 2015). The deep water case describes open-ocean Langmuir turbulence, absent any effects associated with bottom boundary layer shear (Smith et al. 2005). However, when the bathymetry changes across the shelf slope into the coastal zones (i.e., shallow water conditions), there is additional shear associated with the bottom-boundary layer that alters the spatial distribution of turbulent stresses and, in doing so, alters the kinematic properties of LC (Gargett et al. 2004; Gargett and Wells 2007; Tejada-Martínez and Grosch 2007; Tejada-Martínez et al. 2012; Shrestha et al. 2017b). In coastal regions, the height of Langmuir cells is capped by the column depth, and LCs are forced not only by aerodynamic loading in the upper mixed layer but also by the bottom-boundary layer and any ambient pressure gradient. These full-depth Langmuir cells are referred to as Langmuir supercells (Gargett et al. 2004).

Given the pivotal role of Langmuir turbulence in coastal mixing and vertical transport of mass, momentum, and temperature, it is critical to fully understand the cause-and-effect dynamics between forcing conditions and the spatial extent and intensity of Langmuir cells. However, these important LC scales are not well understood (Thorpe 2000). In the literature, efforts to understand these scales of LC were mostly based on field 

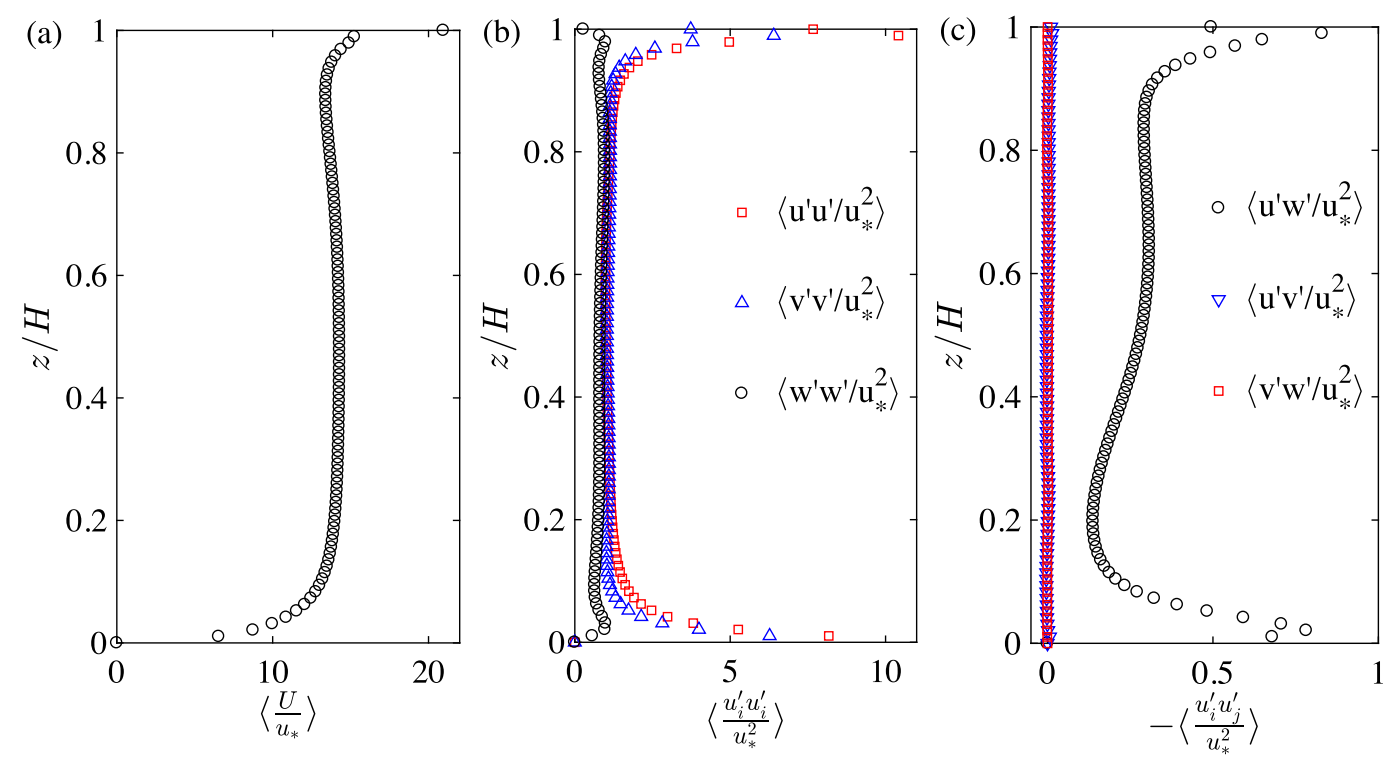

FIG. 6. Time- and horizontally averaged vertical profiles (averaged every two grid points) of the flow referring to case $C_{U_{s}(1), u_{w}(1), k H(1), N(1), L(1)}$ (a) mean velocity, (b) resolved Reynolds normal stresses, and (c) resolved Reynolds shear stresses.

observations. Here, we present a numerical study investigating the factors affecting these LC scales. The advantage of a numerical study is that individual parameters can be systematically varied while others are held constant, thereby isolating the influence of each individual parameters.

\section{a. Langmuir cell width}

Cell width is the horizontal size of a Langmuir cell, which is about half the spacing between two adjacent windrows (see Fig. 1). Researchers have attempted to quantify this length with a nondimensional parameter $\left(L_{s} / H\right)$, which is the ratio of streak spacing (horizontal size of LC) to water depth. Typically in open-ocean scenarios, $L_{s} / H \sim 3-4$ (Smith et al. 1987; Weller and Price 1988). In coastal regions, different values of $L_{s} / H$ have been reported: (i) $\sim 4-5$ (Van Straaten 1950), (ii) $\sim 10$ (Hunter and Hill 1980; Marmorino et al. 2005), (iii) 3-6 (Gargett and Wells 2007), and (iv) 4 (Tejada-Martínez and Grosch 2007; Tejada-Martínez et al. 2012). Unlike the open-ocean case where mixing layer depth $D_{m}$ confines the LC spacing (Smith 1992; Kukulka et al. 2009), coastal LC is capped by the water column depth and thus increases problem complexity. Following the discovery of LSC at the New Jersey coast, there were many open questions on coastal LC that needed to be addressed. Gargett and Wells (2007) posed one open question: What controls $L_{s} / H$ ?

In the literature, some of the field observations have found that this parameter is a function of the atmospheric wind speed (Katz et al. 1965; Zedel and Farmer 1991; Graham and Hall 1997), based on a comparatively small dataset, although there are counterstatements that it is independent of wind speed (Scott et al. 1969). Also, Faller and Caponi (1978) attempted to establish an empirical relation between cell spacing and wavelength of surface waves. Thus, in order to address questions on horizontal extent of "quasi steady" LC, we have performed a set of controlled numerical experiments summarized in Table 1.

Figure 7 shows the dependence of cell spacing on Stokes drift surface velocity and wind stress. Figure $7 \mathrm{~b}$ shows case $C_{U_{s}(1), u_{w}(1), k H(1), N(1), L(1)}$, which is the same flow described in detail in section $3 \mathrm{~b}$. The flow accommodates two pairs of counterrotating LSC, with $L_{s} / H \sim 3$ agreeing with the field observations by Gargett and Wells (2007). For flow visualizations, we have used the $Q$ criterion vortex identifier, which is derived from the velocity gradient tensor, $\mathbf{D}=\nabla \tilde{\mathbf{u}}$ (Hunt et al. 1988; Jeong and Hussain 1995; Christensen and Adrian 2001; Haller 2005). The D can be decomposed into its symmetric and antisymmetric components, $\mathbf{D}=\mathbf{S}+\mathbf{O}$, where $\mathbf{S}=(1 / 2)\left(\nabla \tilde{\mathbf{u}}+\nabla \tilde{\mathbf{u}}^{\mathrm{T}}\right)$ and $\mathbf{O}=(1 / 2)\left(\nabla \tilde{\mathbf{u}}-\nabla \tilde{\mathbf{u}}^{\mathrm{T}}\right)$, allowing computation of the $Q$ criterion with

$$
Q=\frac{1}{2}\left(|\mathbf{O}|^{2}-|\mathbf{S}|^{2}\right)>0
$$

In addition, the spatial isocontours of $Q$ are given the sign of streamwise vorticity; that is, $Q \rightarrow Q \tilde{\omega}_{x} /|\tilde{\boldsymbol{\omega}}|$, which 

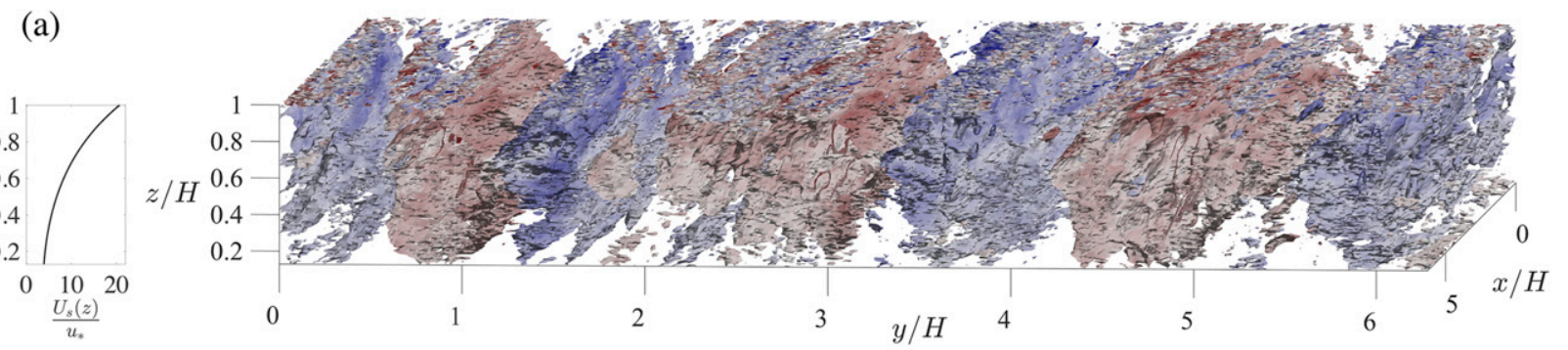

Increased Stokes drift surface velocity

(b)
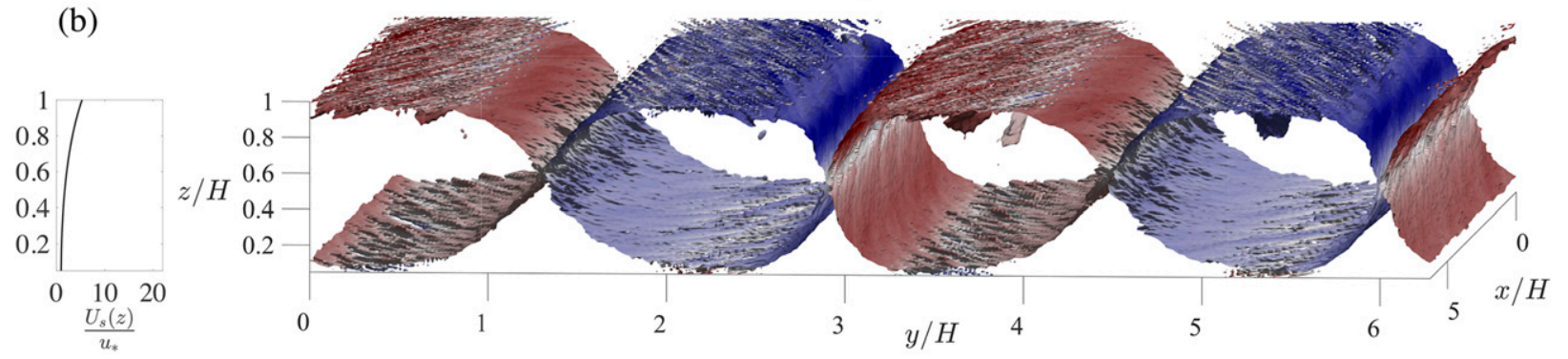

Decreased surface wind stress

(c)
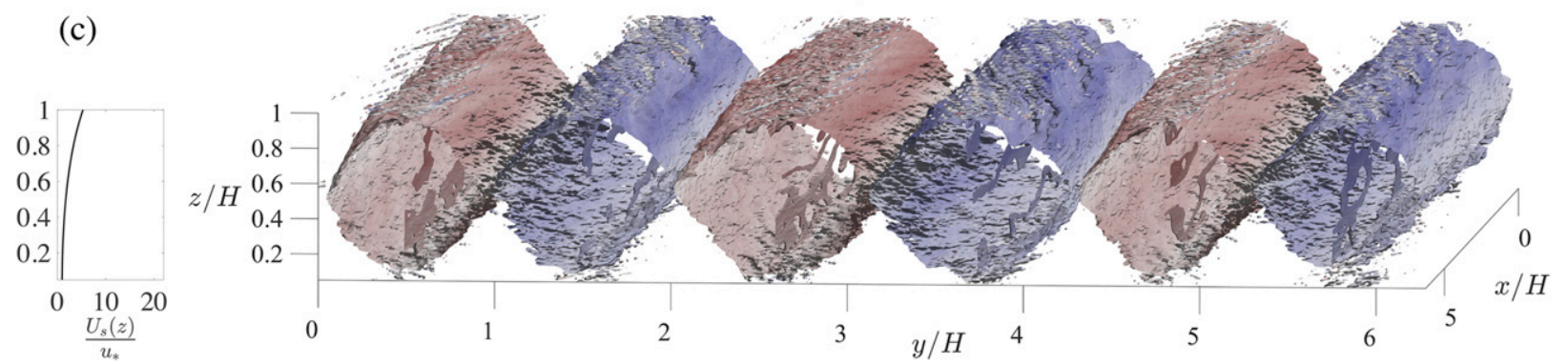

FIG. 7. Three-dimensional isosurfaces for the $Q$ criterion signed by streamwise vorticity. Blue color represents negative streamwise vorticity while red color represents positive streamwise vorticity. Case (a) $C_{U_{s}(2)}$, (b) $C_{U_{s}(1), u_{w}(1), k H(1), N(1), L(1)}$, and (c) $C_{u_{w}(2)}$. The figure depicts the influence of Stokes drift surface velocity magnitude and surface wind stress on Langmuir cell width. Subplot on the left shows the Stokes drift velocity profile.

provides further illumination on the vortical nature of the Langmuir cells.

When only the magnitude of Stokes drift surface velocity was increased from $U_{s} / u_{*}=4.34$ [Fig. $7 \mathrm{~b}$; case $C_{U_{s}(1), u_{w}(1), k H(1), N(1), L(1)}$ ] to $U_{s} / u_{*}=16.95$ [Fig. 7a; case $\left.C_{U_{s}(2)}\right]$ while keeping all the other parameters fixed (here $\mathrm{La}_{t}$ changes from 0.48 to 0.24 while $k H=1.2$ or $\Lambda / H \sim 5$ is fixed), the population of LC increased in the same domain space, resulting in a decreased cell spacing. Note that $\mathrm{La}_{t}$ ranges from 0.2 to 0.8 in the coastal ocean, unlike the open ocean where $\mathrm{La}_{t}$ concentrated around 0.3 (Belcher et al. 2012). The ratio $L_{s} / H$ was found to be in the range 1.5-2.5. This establishes that cell spacing is dependent on the magnitude of Stokes drift surface velocity for fixed $k H$.

In another numerical experiment, only surface wind stress was decreased from $u_{w} / u_{*}=1$ [Fig. $7 \mathrm{~b}$; case
$C_{U_{s}(1), u_{w}(1), k H(1), N(1), L(1)}$ ] to $u_{w} / u_{*}=0.65$ [Fig. $7 \mathrm{c}$; case $C_{u_{w}(2)}$, keeping all the other parameters fixed (here $\mathrm{La}_{t}$ changes from 0.48 to 0.38 ). It resulted in the reduction of horizontal cell width and the ratio $L_{s} / H \sim 2-2.5$ was attained. This shows that imposed surface wind stress is also a parameter controlling LC, conforming with the field observations by Katz et al. (1965), Zedel and Farmer (1991), and Graham and Hall (1997).

Here, we want to emphasize that Stokes drift surface velocity $U_{s} / u_{*}$ and surface wind stress $u_{w} / u_{*}$ individually affect LC spacing. The volume of fluid significantly affected by wind stress is concentrated near the surface, while the Stokes drift velocity is (can be) distributed over the entire water column. Therefore, Stokes drift surface velocity requires a comparatively greater change in magnitude to enhance vertical gradients in the flow. 

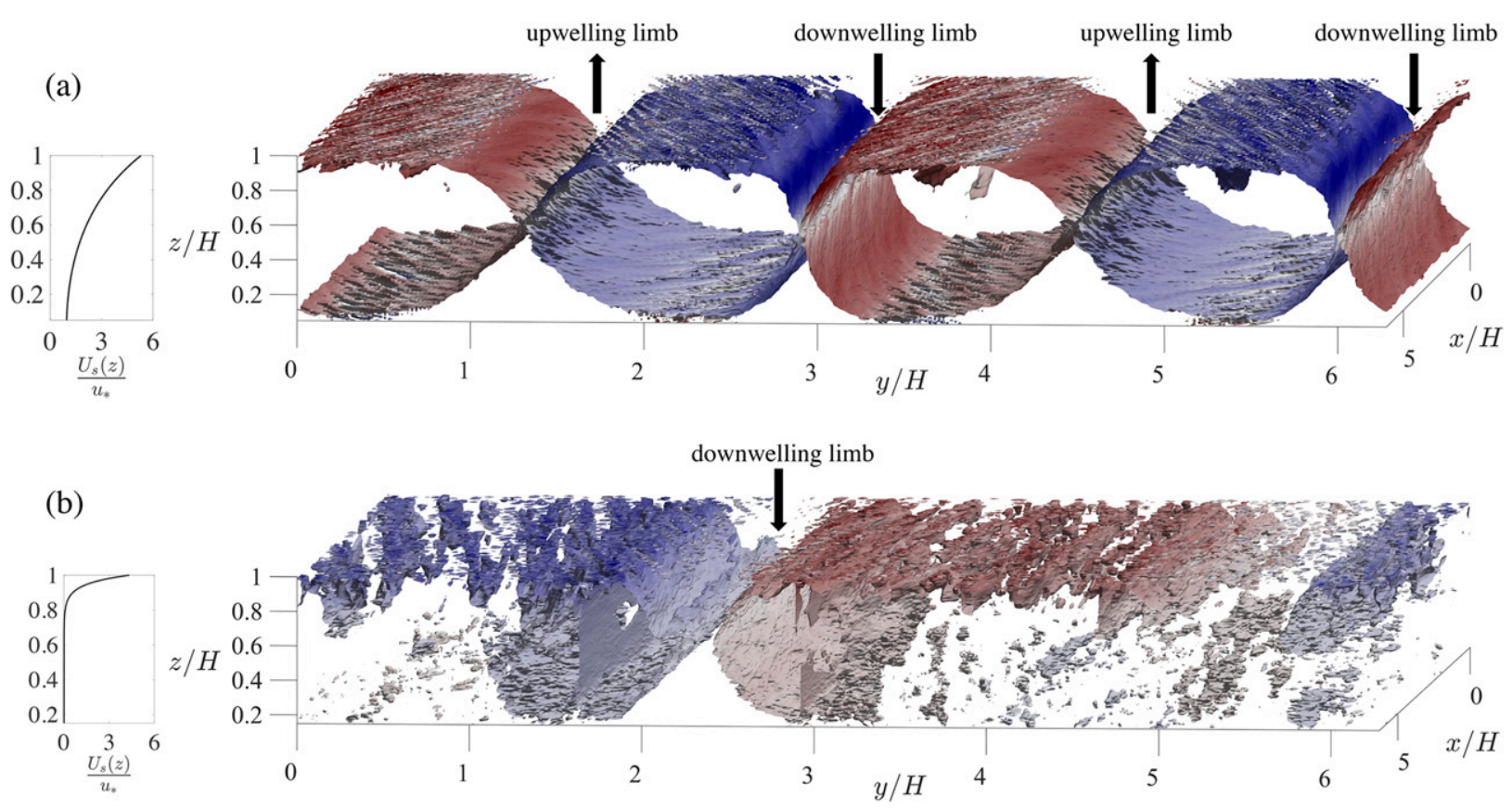

FIG. 8. Three-dimensional isosurfaces for the $Q$ criterion signed by streamwise vorticity. Blue color represents negative streamwise vorticity while red color represents positive streamwise vorticity. Case (a) $C_{U_{s}(1), u_{w}(1), k H(1), N(1), L(1)}$ and (b) $C_{k H(2)}$. The figure depicts the influence of wavenumber on LC height and also LC width. Subplot on the left shows respective Stokes drift velocity profile.

Stokes drift surface velocity magnitude is a representative parameter to the Stokes drift vertical shear, which globally impacts LC structure. Stokes drift vertical shear triggers disturbances to initiate structural modifications and consequently decreases cell spacing. Our study shows that the conditions required for significant structural modifications are the following: (i) $u_{w} / u_{*}<1$ or (ii) $U_{s} / u_{*}>16$. In addition to simulation cases in Table 1 , supplementary simulations listed in Table C1 (in appendix C) have been used to reach this conclusion. In the context of a turbulent Langmuir number, $\mathrm{La}_{t} \lesssim 0.38$ is a necessary, but not sufficient, condition to observe decreasing cell spacing. Similar attempts to study the effect of $\mathrm{La}_{t}$ on Langmuir turbulence were performed by Tejada-Martínez and Grosch (2007) and Kukulka et al. (2011). Tejada-Martínez and Grosch (2007) varied $\mathrm{La}_{t}$ from 0.7 to 0.4, while Kukulka et al. (2011) considered two cases with $\mathrm{La}_{t}=0.78$ and $\mathrm{La}_{t}=0.52$, but they did not observe significant decrease in cell spacing because all the values of $\mathrm{La}_{t}$ were still greater than 0.38 .

While Stokes drift surface velocity and surface wind stress both play significant role in changing Langmuir cell spacing, our study shows that wavenumber also influences the increment in cell spacing as seen in Fig. 8, agreeing with the experiments of Faller and Caponi (1978). Here we make a point only on the horizontal size of LC due to wavenumber (vertical size will be addressed separately in section $4 \mathrm{~b}$ ). In this numerical experiment, only wavenumber was increased from $\mathrm{kH}=$ 1.2 [Fig. 8a; case $C_{U_{s}(1), u_{w}(1), k H(1), N(1), L(1)}$ ] to $k H=10$ [Fig. 8b; case $C_{k H(2)}$ ], keeping all the other parameters fixed (here $\mathrm{La}_{t}$ does not change). It was observed that spacing between the windrows increased and $L_{s} / H \sim 6.3$ was obtained (considering $H$ to be the depth of the water column). Our study shows that one of the conditions necessary to observe increasing cell spacing is $k H \sim 10$. Tejada-Martínez and Grosch (2007) and TejadaMartínez et al. (2012) also performed a similar study by changing $\Lambda$ from $6 H$ to $4 H / 3$, corresponding with $k H=1$ and 4.7 , respectively. Since $k H$ was less than the established necessary condition, $k H \sim 10$, they did not observe changes in cell spacing.

\section{b. LC height}

In general, the forcing that sustains Langmuir circulations is predicated upon Stokes drift via $\mathbf{f}_{L}=\mathbf{u}_{s} \times \tilde{\boldsymbol{\omega}}$, and thus it can be anticipated that the Langmuir cells penetrate until the Stokes depth $\delta_{s}$. Stokes depth is defined as the depth over which Stokes drift profile has nonzero magnitude. However, in the early years, it was believed otherwise; that is, that the Stokes depth did not significantly influence the turbulence dynamics in the ocean surface boundary layer (OSBL). In support of this idea, Li and Garrett (1997) and Grant and Belcher (2009) argued that turbulent kinetic energy (TKE) production due to vortex forcing (vortex forcing generates 
Stokes drift shear limited to shallow near surface regions) was independent of the OSBL depth $h_{*}$, and therefore $k h_{*}$ ( $k$ is wavenumber associated with Stokes decay length) was not considered a suitable scale to represent OSBL turbulence dynamics. This idea was further supported by Polton and Belcher (2007), who provided qualitative evidence that the downwelling jets penetrate beyond the Stokes depth, to the Ekman depth in the context of unstratified open-ocean scenarios with Coriolis accelerations.

These assumptions were countered by the studies of Li et al. (2005), who claimed that the vertical length scale of vertical velocity variance was controlled by $\delta_{s}$, showing how mixed layer averaged vertical velocity variance decreased by $25 \%$ when $\delta_{s}$ was halved. Harcourt and D'Asaro (2008) further extended the analysis in pure wind seas to prove that mixed layer averaged vertical velocity variance is not only a function of $\delta_{s}$, but also $\mathrm{La}_{t}$. Similar results-that is, $\delta_{s}$ is responsible in setting $h_{*}$ or vertical length scales of LC scale with $(k h *)^{-1}$ in the open-ocean settings-were presented by Kukulka and Sullivan (2013), Kukulka and Brunner (2015), and Kukulka and Harcourt (2017).

Does similar scaling hold in the coastal-ocean settings, where bottom boundary shear alters the kinematic properties of LC? Moreover, the coastal LC problem is further simplified by usage of nonrotational LES validated through theoretical analysis by Grosch and Gargett (2016). Therefore, in this regard, does wave forcing absent Coriolis effects have any influence on the vertical length scale of coastal LC? In the past, TejadaMartínez et al. (2012), Sinha et al. (2015), Akan et al. (2013), and Tejada-Martínez et al. (2013) have tried to understand the effect of wave forcing on coastal LC by limited variation of the wavelength of surface waves $\Lambda$ from $6 H$ to $1.3 H$ with $\mathrm{La}_{t}=0.7$ (or $k H=1$ and 4.7, respectively). They remarked that a decrease in $\Lambda$ leads to less coherent full-depth LC, which is characterized by a decline in averaged velocity fluctuation magnitudes. They reported that decreasing wavelength from $6 H$ to $1.3 H$ increased the Stokes drift shear near the surface and intensified small-scale vortices. However, they did not report anything on the vertical scale of LC or the near-surface small-scale eddies.

As a step up to the previous visualization techniques (vertical and crosswind velocity fluctuations, Lumley invariants of the Reynolds stress anisotropy tensor) used by Tejada-Martínez and Grosch (2007), TejadaMartínez et al. (2012), Sinha et al. (2015), Akan et al. (2013), and Tejada-Martínez et al. (2013), we have used three-dimensional isosurfaces for $Q$ criterion, signed by streamwise vorticity. This is a convenient means to assess Langmuir turbulence structures and is explained with Fig. 8. For a flow with low wavenumber $(k H=1.2)$ where the Stokes forcing has nonzero magnitude until the bottom of water column, there exists full-depth LC, as seen in Fig. 8a. When the wavenumber of the surface waves is increased to 10 (Fig. 8b), increases in near-surface Stokes shear leads to intensification of small-scale eddies and the upwelling limb is wide and less coherent. This is exactly a three-dimensional interpretation of the observations reported by TejadaMartínez et al. (2012), Sinha et al. (2015), Akan et al. (2013), and Tejada-Martínez et al. (2013). Further, we point out that the vertical length scale of these smallscale vortices $L_{h}^{\text {ssv }}$ are controlled by the Stokes depth $\delta_{s}$. In Fig. 8 b, $\delta_{s}=0.35 H$ and $L_{h}^{\text {ssv }}$ ranges between $0.25 H$ and $0.35 H$. Therefore, one can conclude that $L_{h}^{\text {ssv }} \sim \delta_{s}$. However, the downwelling limb plunges to the bottom and can be attributed to the presence of bottomboundary layer shear. Unlike the open-ocean case, where Ekman depth controls the penetration depth of downwelling jets, the downwelling limb of coastal LC penetrates to the bottom of the water column for any wavenumber of coastal surface waves $\left(L_{h}^{d}=H\right)$.

Further, the structure of Langmuir turbulence for flows with high and low wavenumber are analyzed with resolved Reynolds normal stress profiles as shown in Fig. 9. The results compare very well with TejadaMartínez et al. (2013), depicting similar effects of wavenumber in the lower portions of the water column. For the low-wavenumber flow represented by case $C_{U_{s}(1), u_{w}(1), k H(1), N(1), L(1)}$, the magnitudes of streamwise, spanwise, and vertical components of Reynolds normal stress are comparable in the middle of the water column, owing to the presence of LC. In flows without LC, the magnitude of Reynolds vertical normal stress at middepth would otherwise be a fraction of Reynolds-averaged streamwise-normal stress. For high wavenumber flow represented by case $\left.C_{k H(2)},\left\langle\tilde{u}^{\prime} \tilde{u}^{\prime}\right\rangle\right\rangle\left\langle\tilde{w}^{\prime} \tilde{w}^{\prime}\right\rangle$ at the lower portions of the water column, $\left\langle\tilde{u}^{\prime} \tilde{u}^{\prime}\right\rangle \approx\left\langle\tilde{w}^{\prime} \tilde{w}^{\prime}\right\rangle$, and $\left\langle\tilde{v}^{\prime} \tilde{v}^{\prime}\right\rangle$ is elevated near the surface. This explains the increase in near-surface Stokes drift shear that intensifies the smallscale LC vortices.

We now demonstrate this flow-high-wavenumber flow where the downwelling limb plunges until the bottom and near-surface small-scale vortices are controlled by the Stokes depth-as Prandtl's secondary flow of the second kind (see Bradshaw 1987). Prandtl's secondary flow of the second kind is sustained by spatial gradients of Reynolds stress components, which causes nonequilibrium in the production and dissipation of TKE and enables mean secondary flow. To prove this, we look into the contours of Reynolds shear stresses and TKE in Fig. 10. Figure 10a clearly depicts the spatial heterogeneity in the distribution of streamwise-vertical 

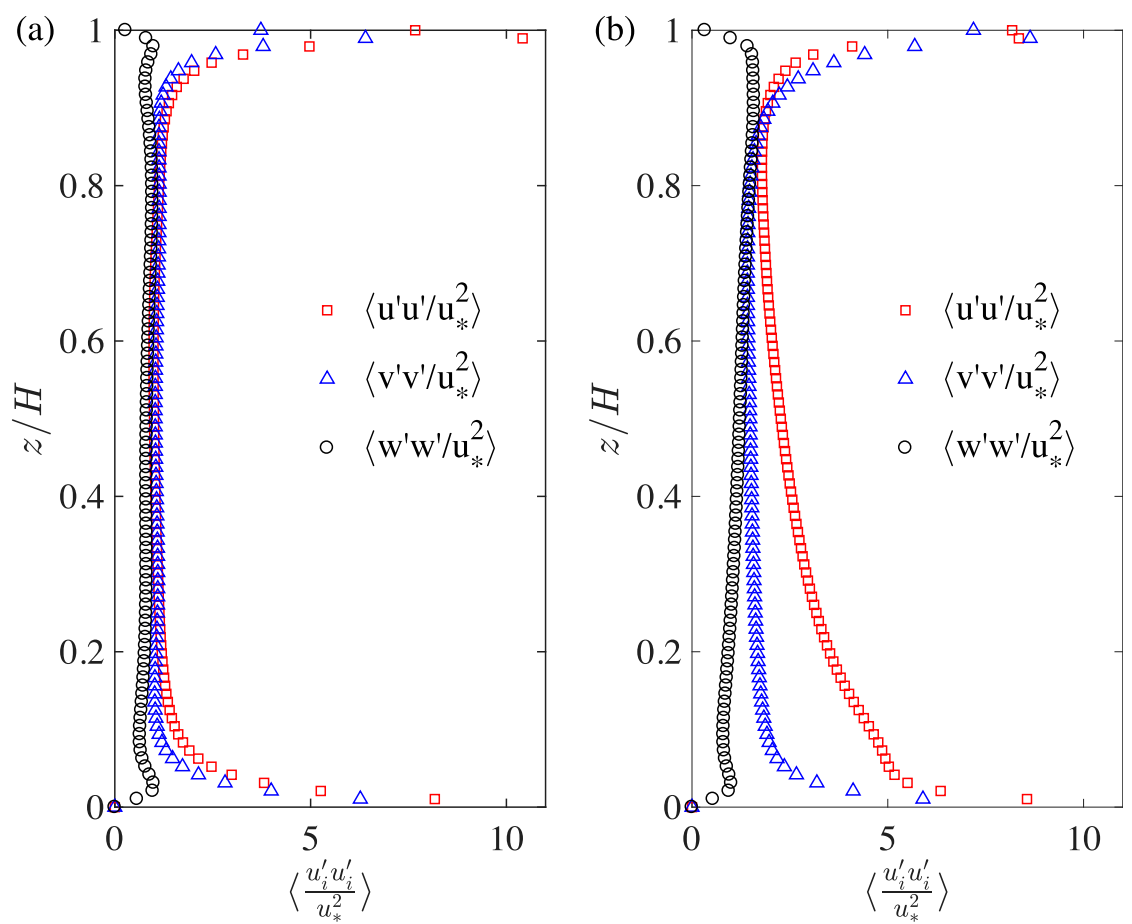

FIG. 9. Time- and horizontally averaged vertical profiles of Reynolds normal stresses (averaged every two grid points). Case (a) $C_{U_{s}(1), u_{w}(1), k H(1), N(1), L(1)}$ and (b) $C_{k H(2)}$.

Reynolds shear stress, which otherwise is not present in a canonical turbulent channel flow. Here, it is easy to understand the highest values of $\left\langle\tilde{u}^{\prime} \tilde{w}^{\prime}\right\rangle$ (high drag) present at the base of the downwelling limb, which is due to the downwelling limb impinging upon the bottom. Also at the location of the upwelling limb, there exists a high value of $\left\langle\tilde{u}^{\prime} \tilde{w}^{\prime}\right\rangle$ due to the presence of positive vertical velocity fluctuations and negative streamwise velocity fluctuations due to the streamwise velocity deficit (Tejada-Martínez and Grosch 2007; see also discussions in section $3 \mathrm{~b}$ ). Figure $10 \mathrm{~b}$ illustrates the spanwise-vertical Reynolds shear stress distribution, which is comparatively lower in magnitude than the other Reynolds shear stress components. This component is primarily responsible for sustaining the secondary flows, and as such large spatial gradients especially at the location of the downwelling limb are clearly seen in the figure. Figure 10c shows the spatial variation of streamwise-spanwise Reynolds shear stress, which is responsible for sustaining secondary flows by laterally exchanging momentum. Finally, Fig. 10d depicts spatial heterogeneity of TKE. High TKE where turbulence production exceeds dissipation is present at the base of the downwelling limb, and low TKE exists just above (middle portion of the downwelling limb). So, this imbalance in production and dissipation of TKE allows turbulence rich fluid to move from the high-TKE region to low-TKE region (Hinze 1967), sustaining the turbulent secondary flow (Anderson et al. 2015).

\section{c. Strength of $L C$}

In the past, the strength of coastal LCs have been quantified in various ways. Gargett et al. (2004) and Gargett and Wells (2007) quantified the strength of downwelling and upwelling limbs through vertical velocity. Later, Tejada-Martínez and Grosch (2007), Tejada-Martínez et al. (2012, 2013), Sinha et al. (2015), Akan et al. (2013), and Walker et al. (2016) adopted fluctuations of crosswind and vertical velocities for quantification. Furthermore, Tejada-Martínez and Grosch (2007), Tejada-Martínez et al. (2013), and Sinha et al. (2015) have performed comparisons of resolved Reynolds stress profiles to measure strength of flows with different $\mathrm{La}_{t}$ and $\Lambda$. Sinha et al. (2015) defined $\left\langle\tilde{w}^{\prime} \tilde{w}^{\prime}\right\rangle_{\mathrm{CC}}^{1 / 2}=\left\langle\left\langle\tilde{w}^{\prime}\right\rangle_{t, x}\left\langle\tilde{w}^{\prime}\right\rangle_{t, x}\right\rangle^{1 / 2}$ (the contribution of coherent cells to the RMS of vertical velocity) as a better method for quantifying strength. Even in other sections of this paper, we have used Reynolds normal stress components, instantaneous velocity contours, and streamwise vorticity to describe the strength of LC. In this section, we introduce compensated circulation, which is directly linked to the magnitude of streamwise-elongated counterrotating vortical LC and thus is another effective measure to quantify strength (Yang and Anderson 2018). 

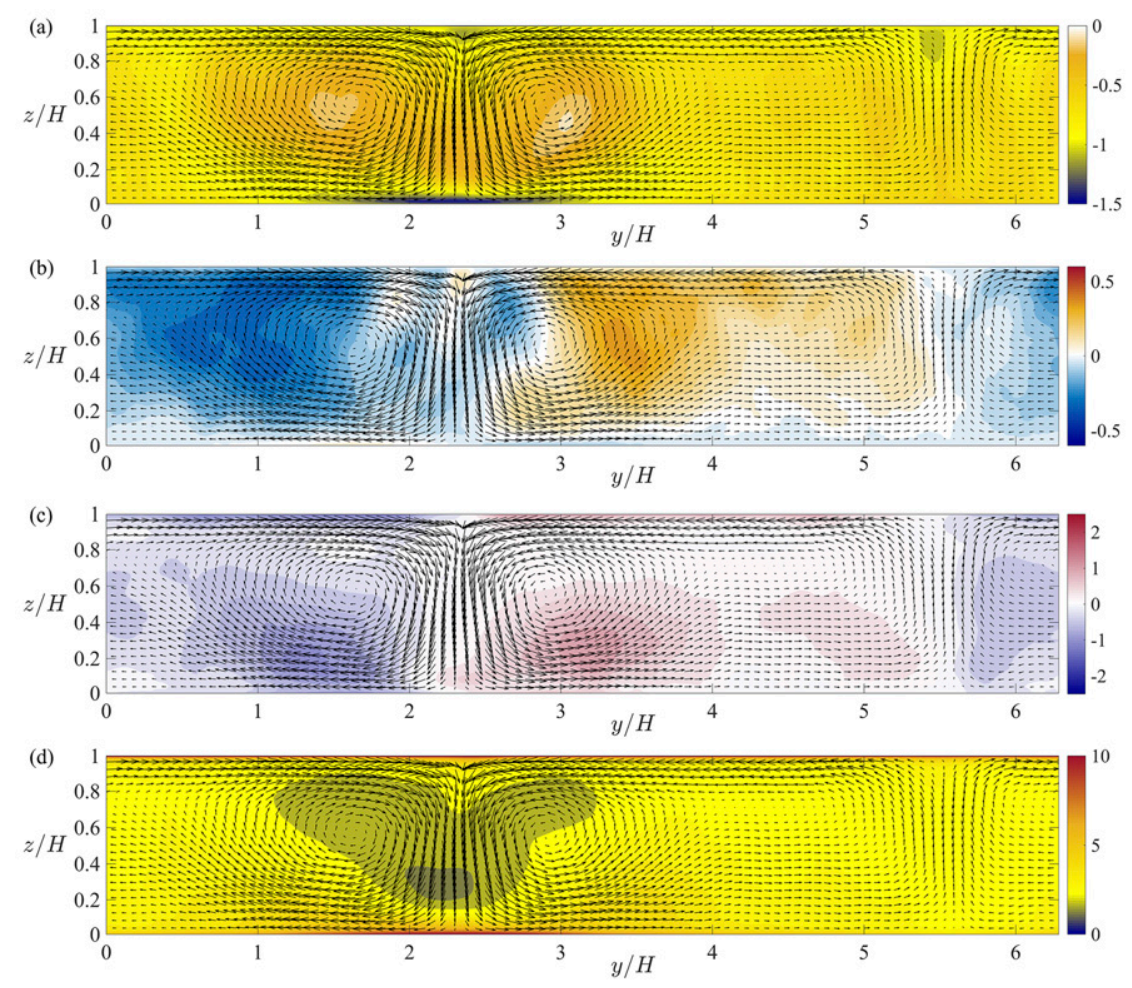

FIG. 10. Reynolds shear stresses and turbulent kinetic energy contours of the flow referring to case $C_{k H(2)}$. (a) Streamwise-vertical Reynolds shear stress, (b) spanwise-vertical component, (c) streamwise-spanwise component, and (d) turbulent kinetic energy.

Compensated circulation per unit area $\Gamma^{*}$, which is based on the absolute value of streamwise vorticity (since the counterrotating vortices would otherwise indicate that circulation is zero), is defined as follows:

$$
\Gamma^{*}=\frac{1}{A} \int_{A}\left|\left\langle\tilde{\omega}_{x}\right\rangle_{x t}(y, z)\right| d A
$$

where $A$ is the area of the spanwise-vertical $(y-z)$ plane.

Figure 11 depicts the quantification of LC intensity through $\Gamma^{*}$, given by Eq. (10) for both the wind-driven flow with wave forcing and colinear wind- and pressuregradient-driven flow with wave forcing. In Fig. 11b $\left(\Gamma^{*}\right.$ vs $\left.U_{s} / u_{*}\right)$, values of $\Gamma^{*}$ vary minimally with $U_{s} / u_{*}$ and thus explain their loose dependence for both types of flows. On the contrary, $\Gamma^{*}$ versus $u_{w} / u_{*}$ (Fig. 11a) exhibits a linear dependence (i.e., $\Gamma^{*} \sim u_{w} / u_{*}$ ) for both types of flows. This conclusion agrees well with the field observations made by Filatov et al. (1981), Harris and Lott (1973), Sutcliffe et al. (1963), and Weller and Price (1988), although their results were based on a fit to scattered data. The field observations made by Gargett and Wells (2007) attained values in rough agreement with the formula $w_{d}^{\prime}=0.008 U_{10}$, predicted by Li and Garrett (1997), where $w_{d}^{\prime}$ is the downward vertical velocity and $U_{10}$ is the wind speed measured at $10-\mathrm{m}$ height. Mechanistically, surface wind stress (spanwise variations of wind results in variable shear force) is the source of vortical structures perpendicular to the ocean surface. These vortices get tilted into the streamwise direction via Stokes drift. Therefore, it is unsurprising that the strength of LC is dependent upon surface wind stress.

\section{Kinematic differences of Langmuir cells in open and coastal zones}

One of the interesting findings from this study is the difference in coastal- and open-ocean Langmuir structures. In Fig. 12, the vertical extent of Langmuir cells in open-ocean settings is dictated by the Stokes/Ekman depth (Figs. 12b,c). However, in coastal zones, Stokes depth only sets the vertical scale of near-surface smallscale vortices, whereas the downwelling limb extends until the bottom for any surface wave forcing (Fig. 12a). This has practical implications and broadens our understanding of vertical transport and mixing processes of materials in coastal- and open-ocean settings.

Note the flow structures of Langmuir turbulence in the open-ocean settings (Figs. 12b,c). Here, the streaks 
(a)

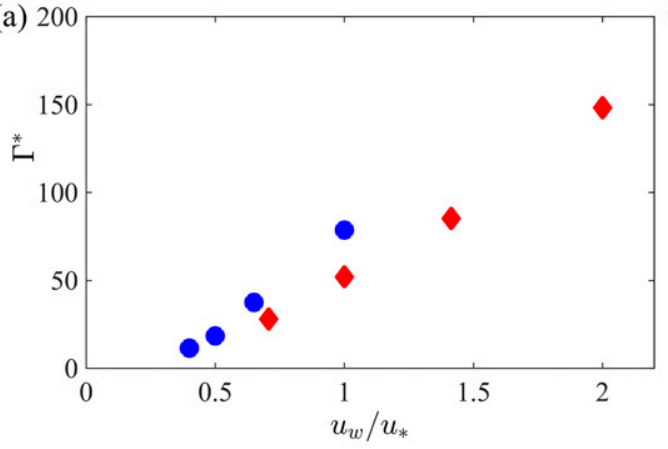

(b)

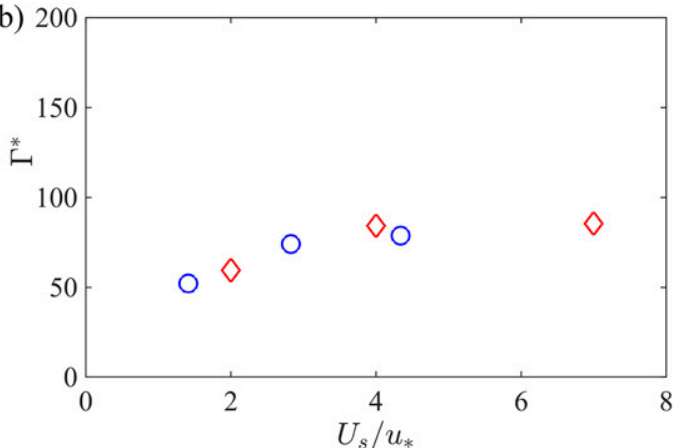

FIG. 11. Compensated circulation as a function of (a) surface wind stress ( $\Gamma^{*}$ vs $u_{w} / u_{*}$ ) and (b) Stokes drift surface velocity $\left(\Gamma^{*} \mathrm{vs} U_{s} / u_{*}\right)$. In each study, both types of flows (i) with pressure gradient forcing (red) and (ii) with no pressure gradient forcing (blue) are considered. Fixed parameters for $\Gamma^{*}$ vs $u_{w} / u_{*}$ with no pressure gradient force: $U_{s} / u_{*}=4.34$ and $k H=1.2$; for $\Gamma^{*}$ vs $u_{w} / u_{*}$ with pressure gradient force: $U_{s} / u_{*}=15$ and $k H=1.2$; for $\Gamma^{*}$ vs $U_{s} / u_{*}$ with no pressure gradient force: $u_{w} / u_{*}=1$ and $k H=1.2$; for $\Gamma^{*}$ vs $U_{s} / u_{*}$ with pressure gradient force: $u_{w} / u_{*}=1.414$ and $k H=1.2$. From the figure, one may conclude that compensated circulation scales linearly with surface wind stress $\left(\Gamma^{*} \sim u_{w} / u_{*}\right)$.

are aligned parallel with one another with finite lateral spacing but are quite irregular. Y junctions-as reported by Li and Garrett (1993)—can also be seen. Distinct differences can be observed in the orientation of Langmuir structures. The disorganization in Fig. 12b is a consequence of Coriolis forcing, wherein the Langmuir structures are aligned in the southeast direction (McWilliams et al. 1997) and are less coherent than the case without Coriolis and buoyancy forcing (Fig. 12c).

Figure 13 compares the Reynolds stress components for coastal- and open-ocean settings [simulation parameters corresponding to Figs. 12a and 12c or cases 2 and 8 (Table 1), respectively, so that they are subjected

(a)

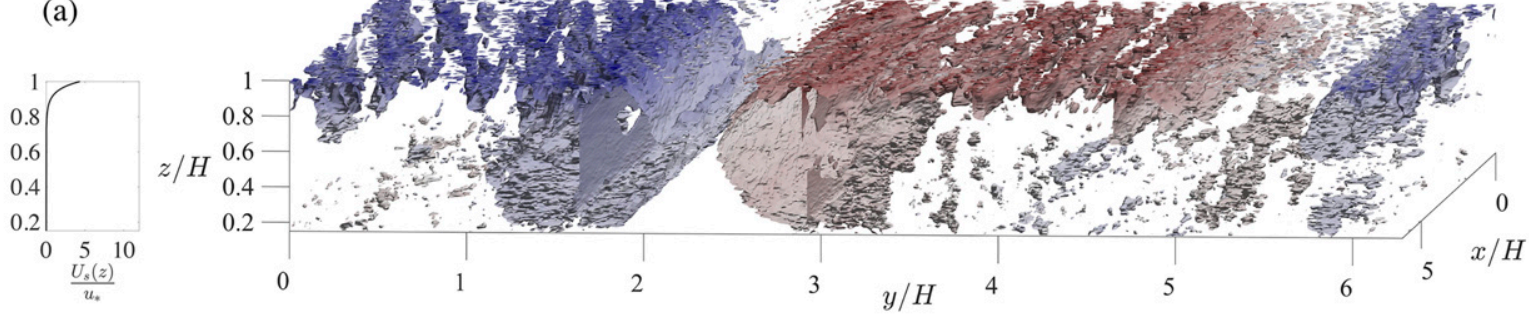

(b)

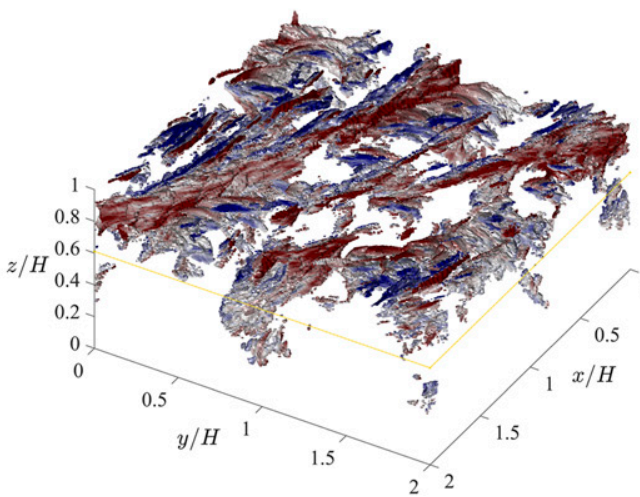

(c)

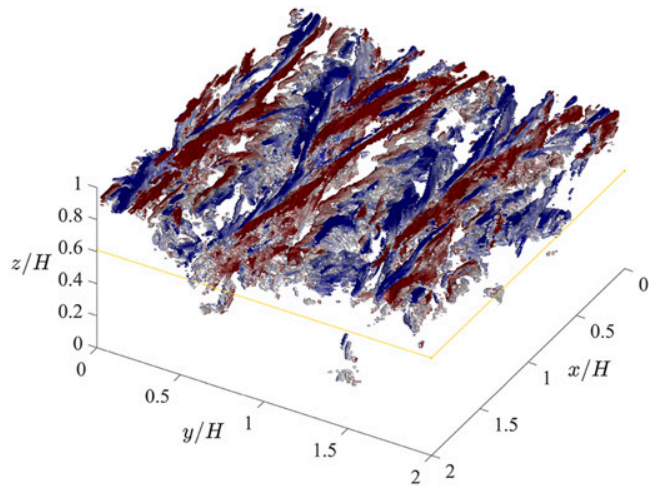

FIG. 12. Comparison of Langmuir structures in open- and coastal-ocean settings with comparable high wavenumber $k H \sim 10$. Threedimensional isosurfaces for the $Q$ criterion signed by streamwise vorticity. Blue color represents negative streamwise vorticity while red color represents positive streamwise vorticity. (a) Coastal zone case, (b) open-ocean case with Coriolis and buoyancy forcing, and (c) open-ocean case with Coriolis and buoyancy forcing nullified. Subplot on the left shows the respective Stokes drift velocity profile. 
(a)

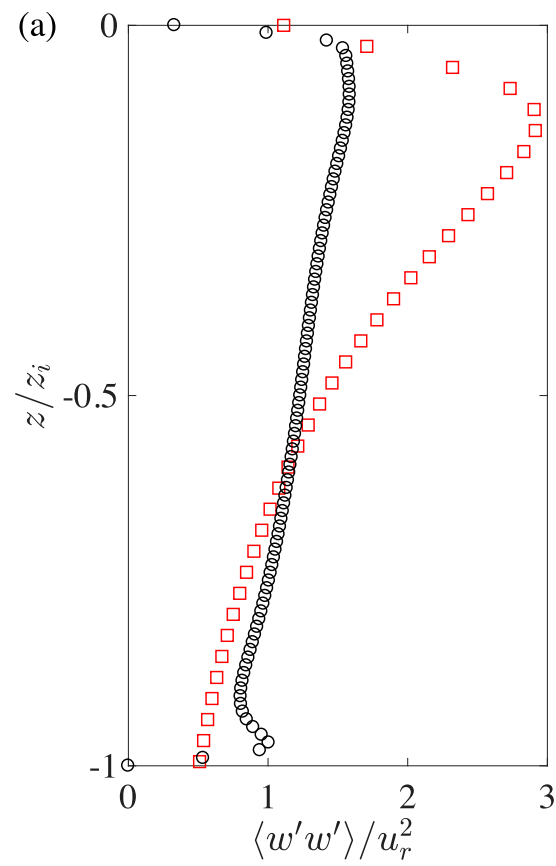

(b)

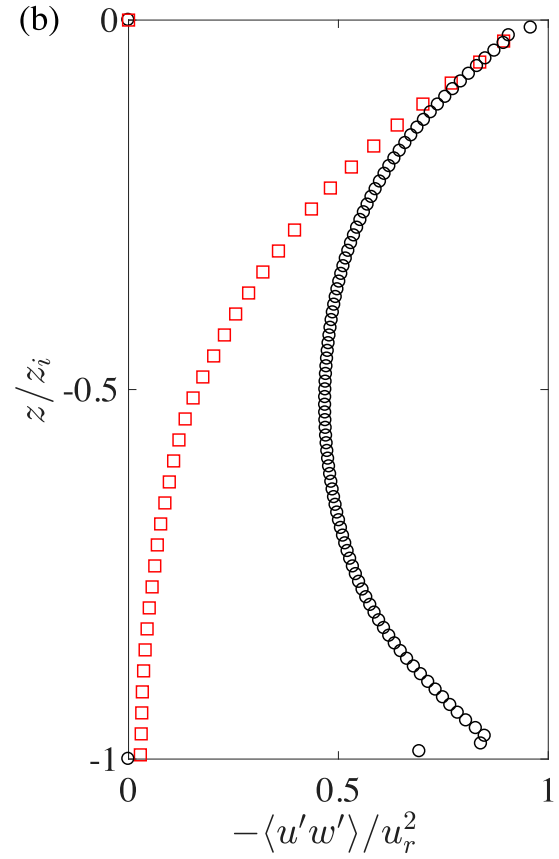

FIG. 13. Time- and horizontally averaged vertical profiles of Reynolds stresses (averaged every two grid points). The vertical axis indicates thermocline depth for the open-ocean case but water-column depth for the coastal-ocean case, and $z=0$ is the water surface. Reynolds stress quantities in the horizontal axis are normalized by respective shear velocity $u_{r}$, which is introduced to have an appropriate scaling for both cases. The $u_{r}$ is surface wind friction velocity for the open-ocean case, but bottom wall friction velocity for the coastal-ocean case. (a) Reynolds vertical normal stresses and (b) Reynolds streamwise-vertical shear stresses. Red squares indicate open-ocean settings $\left(\mathrm{La}_{t}=0.3 ; \Lambda / H=0.66\right)$, and black circles indicate coastal-ocean settings $\left(\mathrm{La}_{t}=0.48 ; \Lambda / H=0.63\right)$.

to similar environmental conditions and a specifically similar Stokes drift profile]. In the figure, respective shear velocity $u_{r}$ is introduced to have an appropriate scaling of Reynolds stresses for both cases. The $u_{r}$ is surface wind friction velocity for the open-ocean case, but bottom wall friction velocity for the coastal-ocean case. The figure tries to explore how under similar vortex forcing (similar Stokes drift profile), the Reynolds stress quantities vary along depth for coastaland open-ocean LC and impact the vertical transport capacity. Comparisons of vertical Reynolds normal stress (Fig. 13a) and streamwise-vertical Reynolds shear stress (Fig. 13b) clearly indicate that the coastal Langmuir cells can exhibit scales far exceeding what would otherwise occur in the open ocean, owing to the role of bottom-boundary shear.

\section{Conclusions}

Comparatively few studies have focused on the coastal Langmuir turbulence, relative to the open ocean. Additionally, the effect of environmental forcing variables on the length scales of LC remains an open question (Gargett and Wells 2007). Here, we consider numerical investigation of LC characteristics in coastal zones by trying to understand the dependency of horizontal and vertical length scales and their structural variations. We explored how prevailing environmental forcing conditions-wind stress, Stokes drift surface velocity, and wavenumber-affect length scales. This was accomplished through a major parametric study, with cases summarized in Table 1. Major findings can be summarized as follows:

1) Our LES results show that decreases in LC spacing are influenced by increasing Stokes drift surface velocity or decreasing surface wind stress, while wavenumber also plays a role in increasing cell spacing. Our LES simulations show that when only the magnitude of Stokes drift surface velocity was increased from $U_{s} / u_{*}=4.34$ to 16.95 , while keeping all the other parameters fixed, the population of LC increased, resulting in a decreased cell spacing. The ratio $L_{s} / H$ was found to be 1.5-2.5. Again, when only 
surface wind stress was decreased from $u_{w} / u_{*}=1$ to 0.65 , while keeping all the other parameters fixed, it resulted in the reduction of horizontal cell width. The ratio $L_{S} / H \sim 2-2.5$ was obtained. Also, when only wavenumber was increased from $k H=1.2$ to 10 , while keeping all other parameters fixed, it was observed that spacing between the windrows increased and the ratio $L_{s} / H \sim 6.3$ was observed.

2) Wavenumber also controls the vertical length scales of LC. For a flow with low wavenumber $(k H=1.2)$, where the Stokes forcing has nonzero magnitude until the bottom of the water column, there exists full-depth LCs. When the wavenumber of the surface waves is increased to 10 , increase in nearsurface Stokes shear leads to intensification of small-scale eddies. The depth scale of these smallscale vortices is controlled by the Stokes depth $\delta_{s}$. However, the downwelling limb of coastal LC extends over the depth of the column for any wavenumber; this is attributed to the presence of bottom-boundary layer shear. Therefore, $L_{h}^{d}=H$ and $L_{h}^{\text {ssv }} \sim \delta_{s}$. We attribute this phenomenon to realization of Prandtl's secondary flow of the second kind.

3) Spanwise variations of wind creates variable surface wind stress, which generates vertical vorticity. These structures are tilted and turned by Stokes drift, aligning them in the direction of the wind. Since wind stress is the main source for the formation of LC, our LES results show linear dependence of LC intensity upon wind stress (quantified with compensated circulation). This agrees with field measurement data provided in preceding studies. It is shown that such characteristics are valid for both wind-driven flows with wave forcing and colinear wind- and pressure-gradientdriven flows with wave forcing.

Acknowledgments. This work was supported by the Texas General Land Office, Oil Spill Program (Program Manager: Steve Buschang) under TGLO Contract 16019-0009283. Computational resources were provided by Texas Advanced Computing Center at The University of Texas at Austin. The authors thank anonymous reviewers for insightful comments, which have improved this manuscript.

\section{APPENDIX A}

\section{Effect of Domain Size and Grid Size}

To verify that our LES results are independent of domain size, we performed simulations with extended horizontal domain size. A similar study has also been carried out by Tejada-Martínez and Grosch (2007) and Golshan et al. (2017). The domain corresponding to Fig. 5 (and all other simulations before this) was of the size $L_{x}=L_{y}=2 \Pi$; therefore, we chose a new set of simulations with domain size $L_{x}=L_{y}=8 \Pi / 3$ [ $\left.\operatorname{case} C_{L(2)}\right]$.
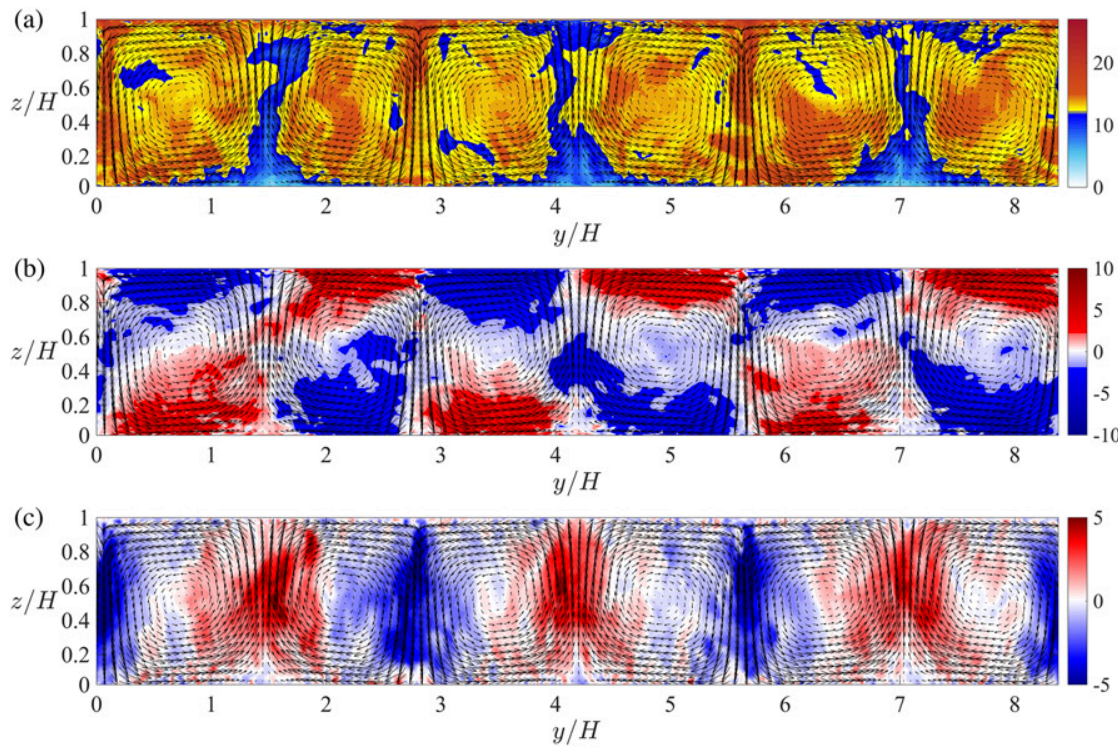

FIG. A1. Instantaneous velocity contours at $L_{x} / 3$ plane of the flow referring to case $C_{L(2)}$ (expanded horizontal domain length: $L_{x}=L_{y}=8 \pi / 3$ ) instantaneous (a) streamwise $\tilde{u}$, (b) spanwise $\tilde{v}$, and (c) vertical $\tilde{w}$. 

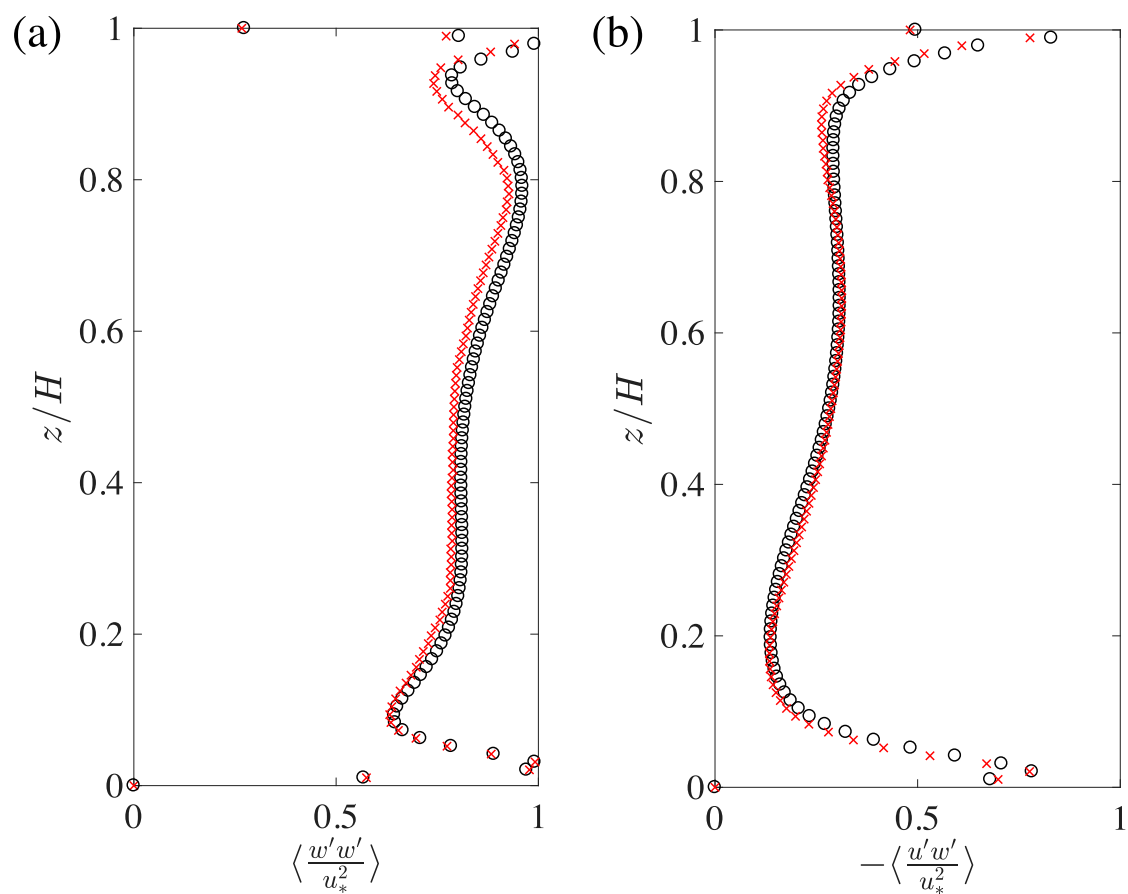

FIG. A2. Time- and horizontally averaged vertical profiles (averaged every two grid points). (a) Reynolds vertical normal stresses and (b) Reynolds streamwise-vertical shear stresses. Black circles indicate case $C_{U_{s}(1), u_{w}(1), k H(1), N(1), L(1)}\left(L_{x}=L_{y}=2 \pi\right)$, and red crosses indicate case $C_{L(2)}$ $\left(L_{x}=L_{y}=8 \pi / 3\right)$.

To preserve grid resolution, the grid size was increased from $192 \times 192 \times 192$ to $256 \times 256 \times 192$.

Figure A1 shows contours of instantaneous streamwise, spanwise, and vertical velocity in the spanwisevertical plane at streamwise location $x=L_{x} / 3$. Figures A1a, A1b, and A1c reveal the instantaneous streamwise, spanwise, and vertical velocity, respectively, displaying the alternating patterns of upwelling and downwelling that are the signature of Langmuir turbulence. The extended domain contains three pairs of counterrotating Langmuir cells, compared to the previous one (Fig. 5 resolved only two pairs of counterrotating Langmuir cells). Therefore, this verified that alternative domain sizes do not affect resultant Langmuir turbulence dynamics. Further, the results for two different domain sizes are compared through vertical profiles of Reynolds vertical normal stress (Fig. A2a) and Reynolds streamwise-vertical shear stress (Fig. A2b). The results exhibit minimal variation between different resolutions.

In another study, Fig. A3 displays the effect of grid size on LES results in the flow with $U_{s} / u_{*}=4.34$, $u_{w} / u_{*}=1$ and $k H=1.2$. Comparisons are made between grid sizes $192 \times 192 \times 192$ [case $\left.C_{U_{s}(1), u_{w}(1), k H(1), N(1), L(1)}\right]$ and $128 \times 128 \times 128$ [case $C_{N(2)}$ ], through Reynolds vertical normal stress (Fig. A3a) and
Reynolds streamwise-vertical shear stress (Fig. A3b) profiles. The figures display negligible difference in the vertical profiles and, thus, establish grid convergence. Therefore, the grid size $192 \times 192 \times 192$ implemented in all the previous simulations is acceptable, and conclusions drawn from simulations performed for this study are not effected by numerical factors.

\section{APPENDIX B}

\section{Colinear Wind- and Pressure-Gradient-Driven Flows with Wave Forcing}

Throughout the paper, we have discussed the importance of Langmuir turbulence for vertical transport and mixing of materials in coastal zones. Additionally, as emphasized in the literature review, most previous coastal LC studies have not considered the effect of pressure gradient force (which is a key contributor to the horizontal transport of oil, sediment, and bioactive materials) assuming insignificant changes in coastal LC characteristics due to prevailing weak tidal velocity (Tejada-Martínez and Grosch 2007; Tejada-Martínez et al. 2013; Sinha et al. 2015). Exceptions are the recent LES simulations by Kukulka et al. (2011), Martinat 

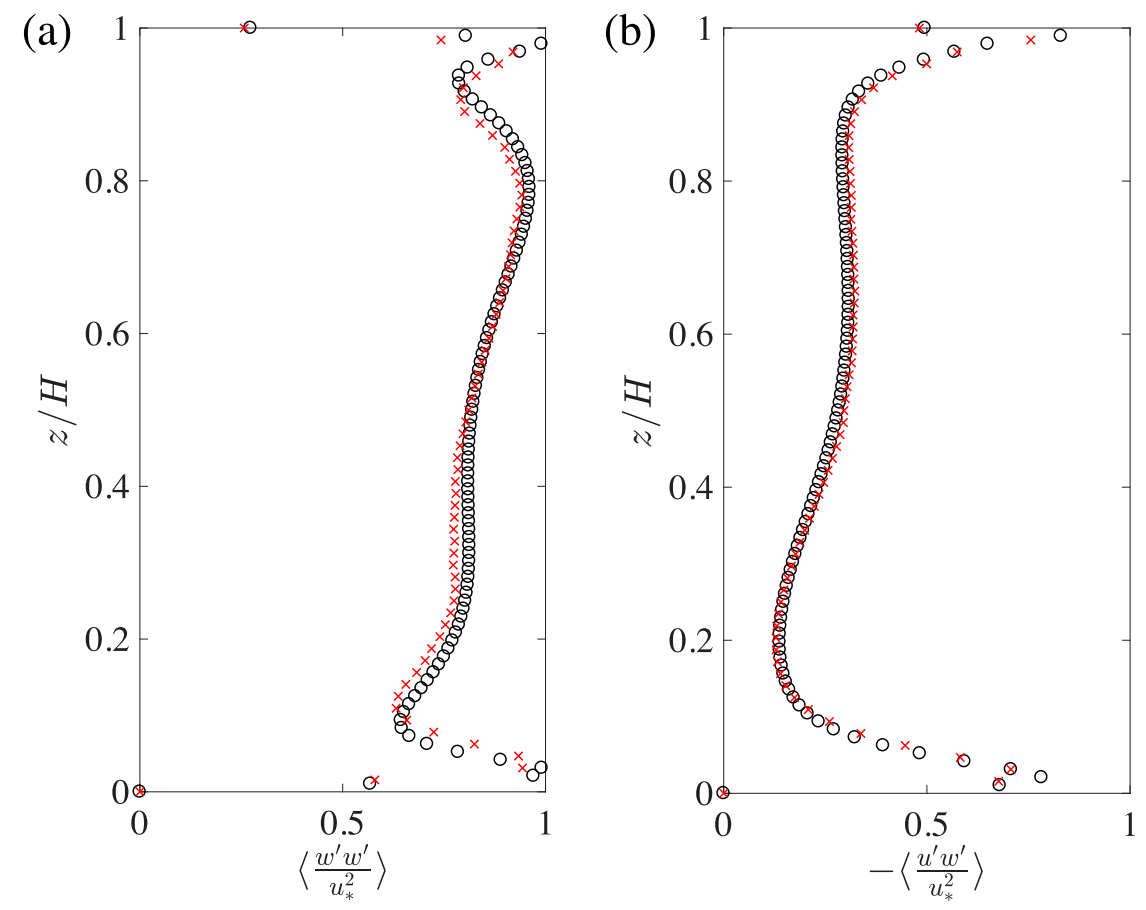

FIG. A3. Time- and horizontally averaged vertical profiles (averaged every two grid points). (a) Reynolds vertical normal stresses and (b) Reynolds streamwise-vertical shear stresses. Black circles indicate case $C_{U_{s}(1), u_{w}(1), k H(1), N(1), L(1)}$ (grid size $\left.192 \times 192 \times 192\right)$, and red crosses indicate case $C_{N(2)}$ (grid size $\left.128 \times 128 \times 128\right)$.

et al. (2011), Li et al. (2013), and Shrestha et al. (2017a, 2018). In this section we explore the effect of downwind pressure gradient force on the length scales of coastal
LC, associating it with large-scale circulations (e.g., due to the Gulf of Mexico's Loop Current; Oye et al. 2005).
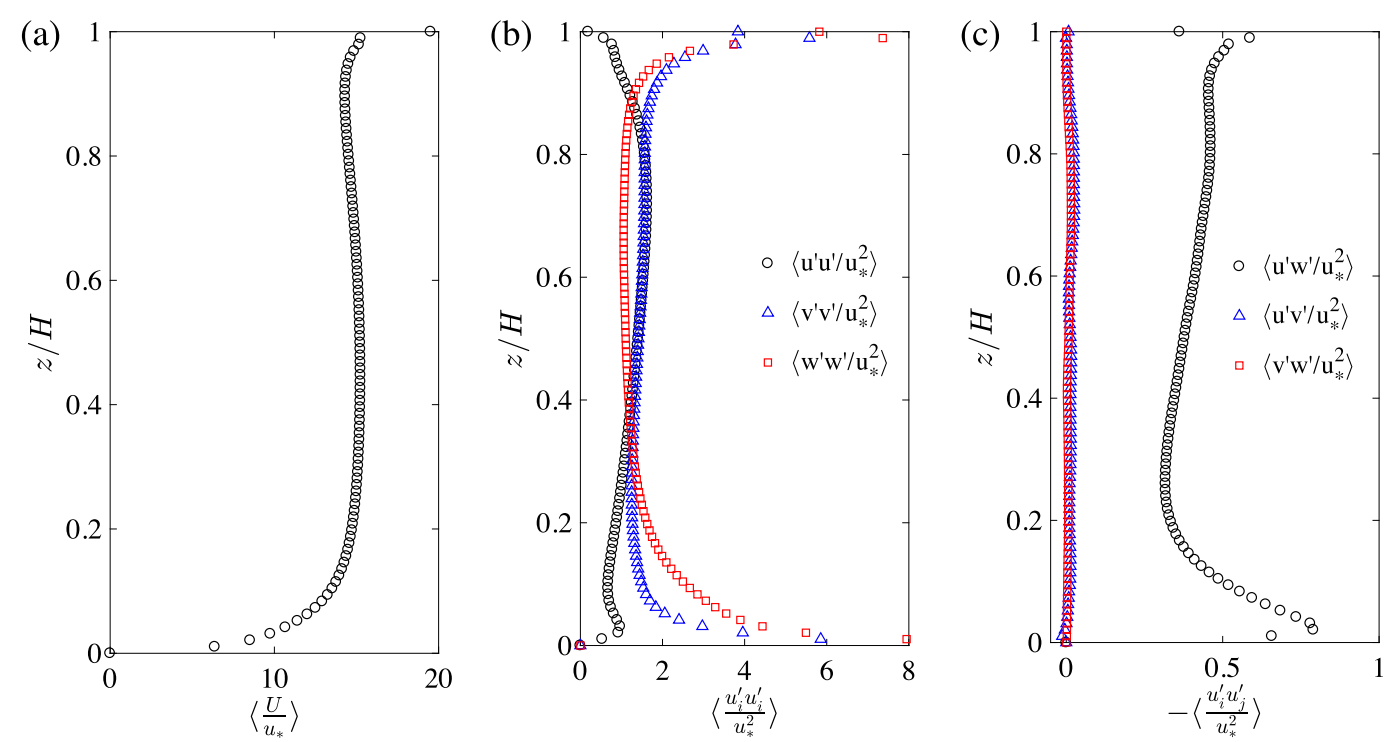

FIG. B1. Time- and horizontally averaged vertical profiles (averaged every two grid points) of the flow referring to case $C \Pi_{U_{s}(1), u_{w}(1), k H(1)}$ (a) mean velocity, (b) resolved Reynolds normal stresses, and (c) resolved Reynolds shear stresses. 
(a)
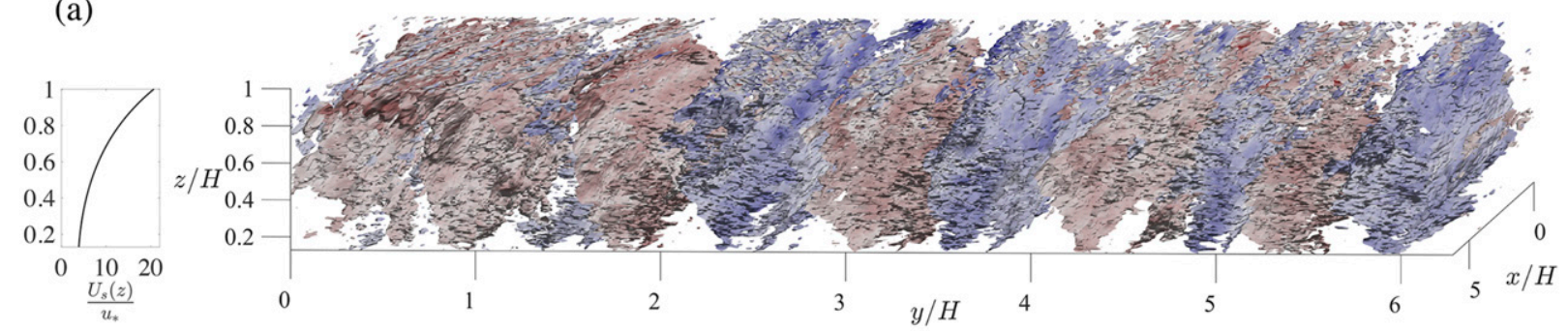

Increased Stokes drift surface velocity

(b)
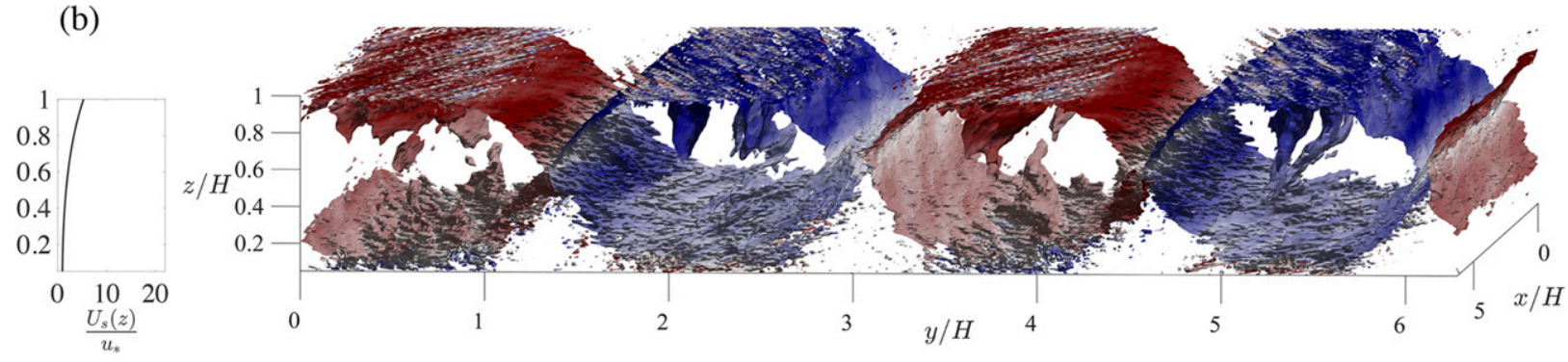

Decreased surface wind stress

(c)
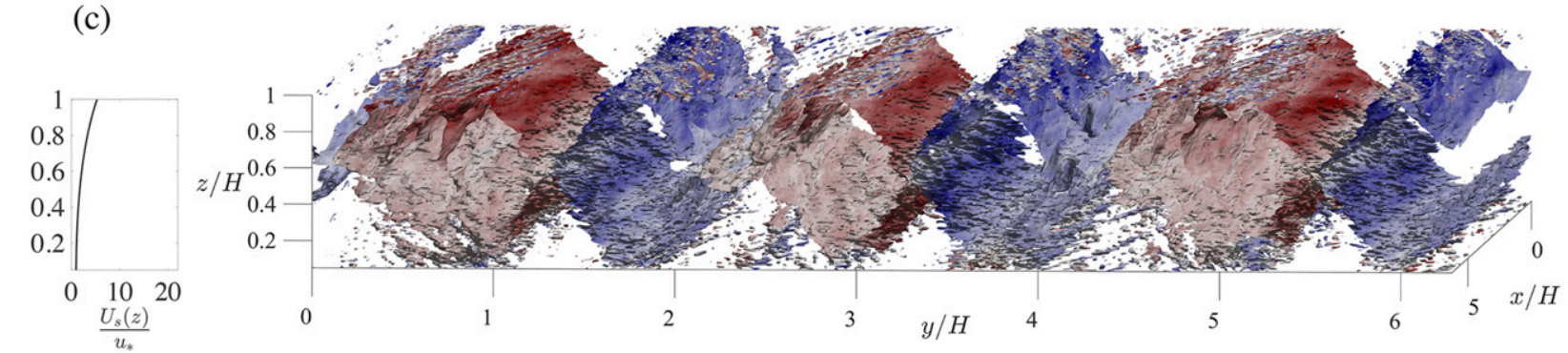

FIG. B2. Three-dimensional isosurfaces for the $Q$ criterion signed by streamwise vorticity. Blue color represents negative streamwise vorticity while red color represents positive streamwise vorticity. Case (a) $C \Pi_{U_{s}(2)}$, (b) $C \Pi_{U_{s}(1), u_{w}(1), k H(1)}$, and (c) $C \Pi_{u_{w}(2)}$. Figure depicts the influence of Stokes drift surface velocity magnitude and surface wind stress on LC width for a pressure-gradient-driven flow. Subplot on the left shows the Stokes drift velocity profile.

For this purpose, forcing parameters similar to the previous setup as described in section $3 b$ have been imposed, except that in the present context the downwind pressure gradient force is imposed. The computational domain is a "half-channel flow," with a rectangular domain of spatial extent $L_{x}=L_{y}=2 \Pi$, depth $H=1$ (or $L_{x}=94.25 \mathrm{~m}, L_{y}=94.25 \mathrm{~m}, H=15 \mathrm{~m}$ ), and computational grid size $192 \times 192 \times 192$. Constant wind stress $0.01 \mathrm{~N} \mathrm{~m}^{-2}$, coaligned with the wave forcing direction, is considered on the top boundary of the shallow water column $(H=15 \mathrm{~m})$. This gives surface friction velocity $u_{w}$ to be $3.22 \times 10^{-3} \mathrm{~m} \mathrm{~s}^{-1}$. Surface waves are characterized by frequency $\sigma_{w}=0.82 \mathrm{rad} \mathrm{s}^{-1}$; amplitude $a \sim 0.67 \mathrm{~m}$; wavenumber $k=0.08 \mathrm{rad} \mathrm{m}^{-1}$ corresponding to wavelength $\Lambda=79 \mathrm{~m}(\Lambda / H=5.26)$, and Stokes drift surface velocity $U_{s}=0.036 \mathrm{~m} \mathrm{~s}^{-1}$. This gives turbulent Langmuir number [Eq. (6) $] \mathrm{La}_{t}=0.3$ and nondimensional wavenumber $k H=1.2$. Since pressure gradient force is imposed in this LES simulation, calculations yield mean current speed $U_{c}=1.3 \mathrm{~m} \mathrm{~s}^{-1}$, using the relation $\tau_{*}=\rho C_{d} U_{c}^{2}$. Here, $C_{d}$ is the drag coefficient equaling $2.5 \times 10^{-3}$ (Sternberg 1968; Sherwood et al. 2006), $\tau_{*}$ is bottom-boundary layer surface stress, and $U_{c}$ is the mean current speed due to the "loop current ring" shed from the Loop Current. The value of $1.3 \mathrm{~m} \mathrm{~s}^{-1}$ falls within the range (peak speed $\sim 1.8 \mathrm{~m} \mathrm{~s}^{-1}$ ) observed by Oye et al. (2005). Also, the ratio of surface wind friction velocity to the bottom friction velocity due to pressure gradient stress is defined as follows: 

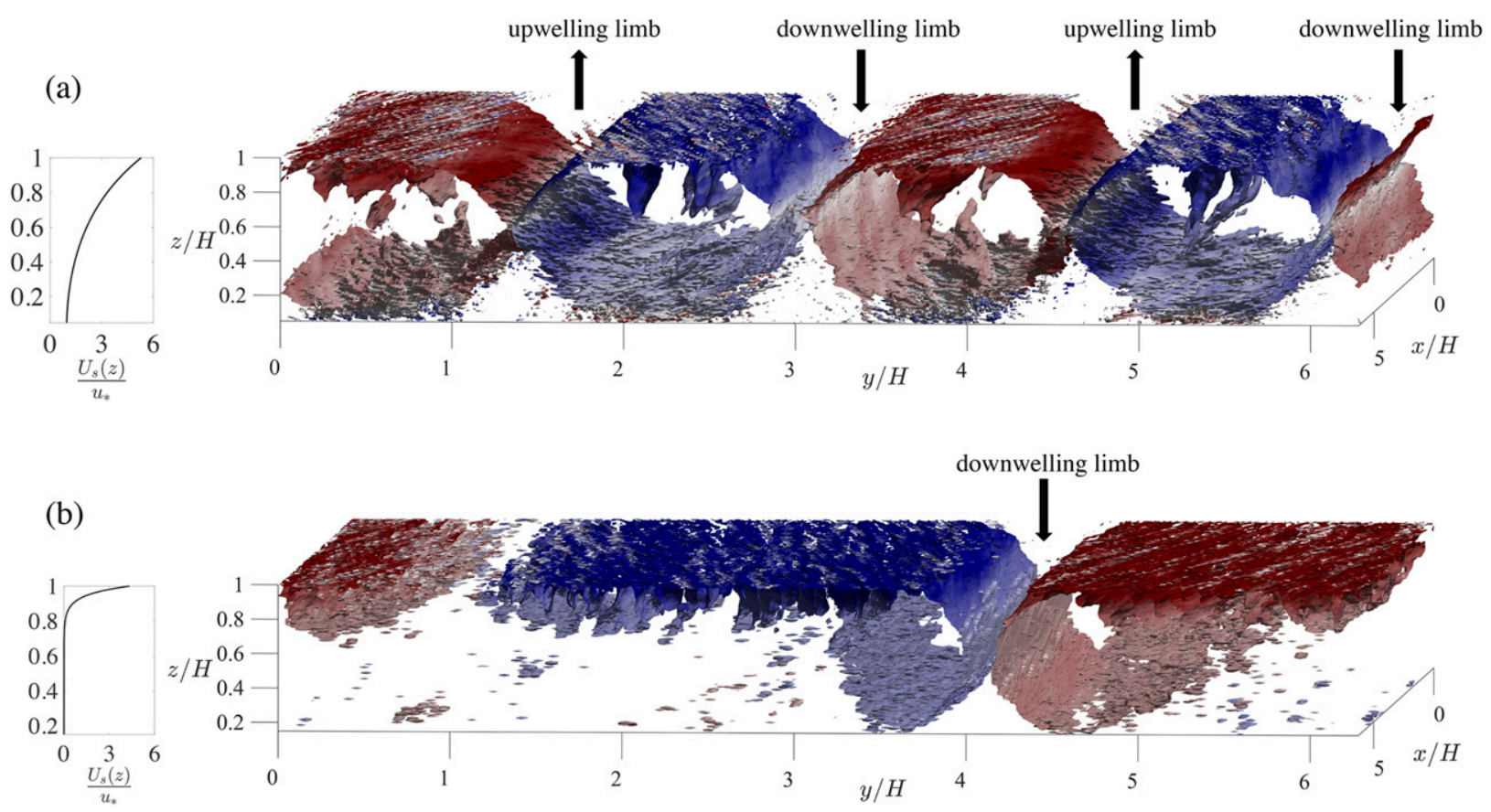

FIG. B3. Three-dimensional isosurfaces for the $Q$ criterion signed by streamwise vorticity. Blue color represents negative streamwise vorticity while red color represents positive streamwise vorticity. Case (a) $C \Pi_{U_{s}(1), u_{w}(1), k H(1)}$ and $k H=1.2$ (or La $\mathrm{a}_{t}=0.3$; $\Lambda / H=5.26$ ) and (b) $C \Pi_{k H(2)}$. The figure depicts the influence of wavenumber on LC height and also LC width for a pressure gradient driven flow. Subplot on the left shows respective Stokes drift velocity profile.

$$
\begin{aligned}
& \lambda=\frac{u_{w}}{u_{*}}=\left(\frac{\tau_{w} \rho_{w}^{-1}}{\tau_{*} \rho_{*}^{-1}}\right)^{1 / 2}=1.414, \quad \text { or } \\
& \Psi=\left(\frac{\tau_{w}}{\tau_{*}}\right)=2,
\end{aligned}
$$

where $u_{w}$ is surface friction velocity, $u_{*}$ is bottom friction velocity, $\tau_{w}$ is top-surface wind stress, and $\tau_{*}$ is bottom-boundary layer surface stress (Martinat et al. 2011; Gargett and Grosch 2014). The $\Psi$ is related to the parameter $\gamma$ used by Martinat et al. (2011), as $\gamma=1 / \Psi=0.5$.

Coriolis and buoyancy forcing have been deactivated, as per the studies by Tejada-Martínez and Grosch (2007), Kukulka et al. (2012), and section 3b. The use of nonrotational LES of LC has also been validated very recently by Grosch and Gargett (2016), through theoretical analysis in the absence of pressure gradient force. However, even when pressure gradient force is activated, omission of Coriolis force is still valid. Here, we follow this approach, to show that we can neglect Coriolis accelerations in the presence of pressure gradient forcing developed by the "Loop Current ring." Conforming to the sketch in Fig. 1 of Grosch and Gargett (2016), we add a pressure gradient force in the $y$ direction (i.e., coaligned with surface wind stress).
Addition of downwind pressure gradient force only modifies Eq. (6) (case A: with rotation and a lateral boundary) and Eq. (10) (case B: no rotation and no lateral boundary) to the following:

$$
\begin{aligned}
& 0=-g\left(\frac{\partial \eta}{\partial y}\right)-\gamma v-\frac{\tau_{0}}{\rho H}, \quad \text { and } \\
& 0=-g\left(\frac{\partial \eta}{\partial y}\right)-\gamma v-\frac{\tau_{0}}{\rho H} .
\end{aligned}
$$

However, the two modified equations are identical. Therefore, time-mean velocities in both cases are still identical, and, thus, Coriolis force can be neglected.

Now, we present the results of the LES simulations and show that the activation of pressure gradient force does not make any significant differences to the length scales of LC, compared to previous simulations without pressure gradient forcing. Figure B1 validates the presence of Langmuir turbulence with the vertical Reynolds stress profiles. Here, the same explanations for Fig. 6 agree well. Figure B2 reveals the effect of increased Stokes drift surface velocity and decreased surface wind stress on the horizontal length scale of LC by reproducing similar three-dimensional isosurfaces for the $Q$ criterion signed by streamwise vorticity, as in Fig. 7. For this, cases $C \Pi_{U_{s}(2)}($ Fig. B2a), 

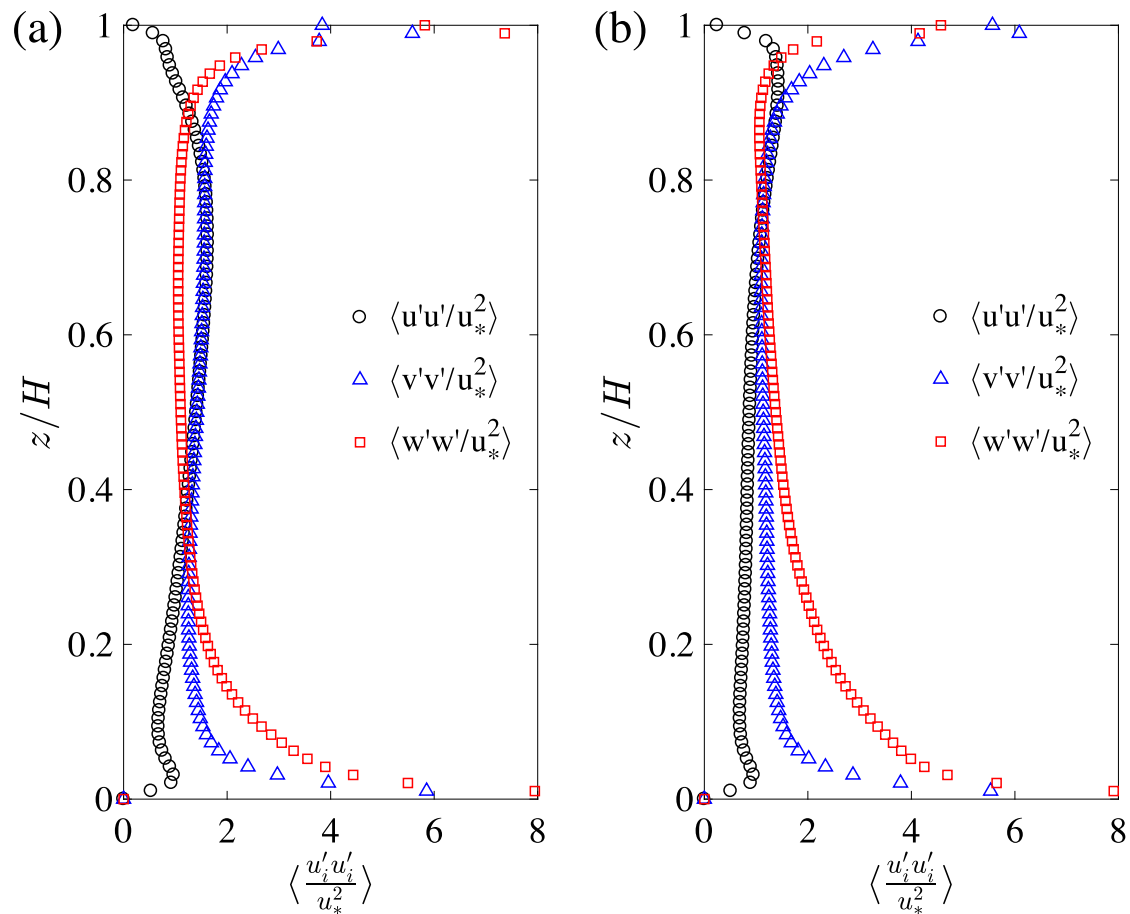

FIG. B4. Time- and horizontally averaged vertical profiles of Reynolds normal stresses (averaged every two grid points) for colinear wind- and pressure-gradient-driven flows with wave forcing. Case (a) $C \Pi_{U_{s}(1), u_{w}(1), k H(1)}$ and (b) $C \Pi_{k H(2)}$.

$C \Pi_{U_{s}(1), u_{w}(1), k H(1)}$ (Fig. B2b), and $C \Pi_{u_{w}(2)}$ (Fig. B2c) were considered. The figure clearly shows no effect of imposed pressure gradient force on horizontal size of LC. Similarly, cases $C \Pi_{U_{s}(1), u_{w}(1), k H(1)}$ (Fig. B3a) and $C \Pi_{k H(2)}$ (Fig. B3b) were considered to understand the effect of wavenumber on the vertical size of LC when forced by a pressure gradient. The results on vertical length scale agreed closely with Fig. 8. Figure B4 supports the above result, displaying similar characteristics of Langmuir turbulence with varying wavenumber, as seen in Fig. 9.

\section{APPENDIX C}

\section{Supplementary Numerical Experiments}

Table $\mathrm{C} 1$ presents a list of supplementary numerical simulations, which supports Table 1 in deducing threshold

TABLE C1. List of supplementary numerical experiments not included in Table 1 to avoid technical congestion but involved in deducing individual threshold conditions required to observe structural modifications (section 4). Note that identifiers are named similarly as in Table 1. Bold values indicate those simulation parameters that are varied while others are fixed. Also, $N^{*}=N_{x}=N_{y}=N_{z}$ and $L^{\dagger}=$ $\left(L_{x}, L_{y}, L_{z}\right)$.

\begin{tabular}{|c|c|c|c|c|c|c|c|c|}
\hline Case & $U_{s} / u_{*}$ & $u_{w} / u_{*}$ & $k H$ & $\Pi$ & Coriolis & Buoyancy & $\operatorname{Grid}\left(N^{*}\right)$ & Domain $\left(L^{\dagger}\right)$ \\
\hline$C_{U_{s}(5)}$ & 8.68 & 1 & 1.2 & 0 & No & No & 192 & $2 \pi, 2 \pi, 1$ \\
\hline$C_{U_{s}(6)}$ & 1 & 1 & 1.2 & 0 & No & No & 192 & $2 \pi, 2 \pi, 1$ \\
\hline$C_{u_{w}(5)}$ & 4.34 & 2 & 1.2 & 0 & No & No & 192 & $2 \pi, 2 \pi, 1$ \\
\hline$C_{u_{w}(6)}$ & 4.34 & 0.32 & 1.2 & 0 & No & No & 192 & $2 \pi, 2 \pi, 1$ \\
\hline$C_{U_{s}(7), u_{w}(7)}$ & 2.83 & 0.65 & 1.2 & 0 & No & No & 192 & $2 \pi, 2 \pi, 1$ \\
\hline$C_{U_{s}(7), u_{w}(7)}$ & 1.41 & 0.32 & 1.2 & 0 & No & No & 192 & $2 \pi, 2 \pi, 1$ \\
\hline$C_{k(3)}$ & 4.34 & 1 & 4.7 & 0 & No & No & 192 & $2 \pi, 2 \pi, 1$ \\
\hline$C_{k(4)}$ & 1.41 & 0.32 & 1.2 & 0 & No & No & 192 & $2 \pi, 2 \pi, 1$ \\
\hline$C \Pi_{U_{s}(6)}$ & 10 & 1.41 & 1.2 & 1 & No & No & 192 & $2 \pi, 2 \pi, 1$ \\
\hline$C \Pi_{u_{w}(5)}$ & 15 & 8 & 1.2 & 1 & No & No & 192 & $2 \pi, 2 \pi, 1$ \\
\hline$C \Pi_{u_{w}(6)}$ & 15 & 6 & 1.2 & 1 & No & No & 192 & $2 \pi, 2 \pi, 1$ \\
\hline$C \prod_{u_{w}(7)}$ & 15 & 4 & 1.2 & 1 & No & No & 192 & $2 \pi, 2 \pi, 1$ \\
\hline
\end{tabular}


conditions required to observe change in LC spacing. As mentioned in section $4 \mathrm{a}$, the threshold conditions for case $C_{U_{s}(1), u_{w}(1), k H(1), N(1), L(1)}$ to observe such changes are (i) $u_{w} / u_{*}<1$ or (ii) $U_{s} / u_{*}>16$.

\section{REFERENCES}

Akan, C., A. E. Tejada-Martínez, C. Grosch, and G. Martinat, 2013: Scalar transport in large-eddy simulation of Langmuir turbulence. Cont. Shelf Res., 55, 1-16, https://doi.org/10.1016/ j.csr.2012.12.009.

Anderson, W., and C. Meneveau, 2011: Dynamic large-eddy simulation model for boundary layer flow over multiscale, fractallike surface. J. Fluid Mech., 679, 288-314, https://doi.org/ 10.1017/jfm.2011.137.

— , and M. Chamecki, 2014: Numerical study of turbulent flow over complex aeolian dune fields: The White Sands National Monument. Phys. Rev., 89, 013005, https://doi.org/10.1103/ PhysRevE.89.013005.

_ J. Barros, K. Christensen, and A. Awasthi, 2015: Numerical and experimental study of mechanisms responsible for turbulent secondary flows in boundary layer flows over spanwise heterogenous roughness. J. Fluid Mech., 768, 316-347, https:// doi.org/10.1017/jfm.2015.91.

Assaf, G., R. Gerard, and A. Gordon, 1971: Some mechanisms of oceanic mixing revealed in aerial photographs. J. Geophys. Res., 76, 6550-6572, https://doi.org/10.1029/JC076i027p06550.

Belcher, S., and Coauthors, 2012: A global perspective on Langmuir turbulence in the ocean surface boundary layer. Geophys. Res. Lett., 39, L18605, https://doi.org/10.1029/ 2012 GL052932.

Bou-Zeid, E., C. Meneveau, and M. Parlange, 2005: A scaledependent Lagrangian dynamic model for large eddy simulation of complex turbulent flows. Phys. Fluids, 17, 025105, https://doi.org/10.1063/1.1839152.

Bradshaw, P., 1987: Turbulent secondary flows. Annu. Rev. Fluid Mech., 19, 53-74, https://doi.org/10.1146/annurev.fl.19.010187.000413.

Chester, S., C. Meneveau, and M. Parlange, 2007: Modelling of turbulent flow over fractal trees with renormalized numerical simulation. J. Comput. Phys., 225, 427-448, https://doi.org/ 10.1016/j.jcp.2006.12.009.

Christensen, K., and R. Adrian, 2001: Statistical evidence of hairpin vortex packets in wall turbulence. J. Fluid Mech., 431, 433-443, https://doi.org/10.1017/S0022112001003512.

Churchill, J., A. Plueddemann, and S. Faluotico, 2006: Extracting wind sea and swell from directional wave spectra derived from a bottom-mounted ADCP. Woods Hole Oceanographic Institution Tech. Rep. WHOI-2006-13, 41 pp., https://doi.org/ 10.1575/1912/1372.

Craik, A., and S. Leibovich, 1976: A rational model for Langmuir circulation. J. Fluid Mech., 73, 401-426, https://doi.org/ 10.1017/S0022112076001420.

D'Alessio, S., K. Abdella, and N. A. McFarlane, 1998: A new secondorder turbulence closure scheme for modeling the oceanic mixed layer. J. Phys. Oceanogr., 28, 1624-1641, https://doi.org/ 10.1175/1520-0485(1998)028<1624:ANSOTC $>2.0 . C O ; 2$.

D'Asaro, E., 2014: Turbulence in the upper-ocean mixed layer. Annu. Rev. Mar. Sci., 6, 101-115, https://doi.org/10.1146/ annurev-marine-010213-135138.

Dyke, P., and S. Barstow, 1983: The importance of Langmuir circulations to the ecology of the mixed layer in North Sea dynamics. North Sea Dynamics, J. Sündermann and W. Lenz, Eds., Springer, 486-497.

Faller, A., and A. Woodcock, 1964: The spacing of windrows of Sargassum in the ocean. J. Mar. Res., 22, 22-29.

— Langmuir circulations. J. Geophys. Res., 83, 3617-3633, https:// doi.org/10.1029/JC083iC07p03617.

— , and S. Auer, 1988: The roles of Langmuir circulations in the dispersion of surface tracers. J. Phys. Oceanogr., 18, 1108-1123, https://doi.org/10.1175/1520-0485(1988)018<1108: TROLCI $>2.0 . \mathrm{CO} ; 2$.

Filatov, N., S. Rjanzhin, and L. Zaycev, 1981: Investigation of turbulence and Langmuir circulation in Lake Ladoga. J. Great Lakes Res., 7, 1-6, https://doi.org/10.1016/S0380-1330(81)72016-1.

Fox-Kemper, B., G. Danabasoglu, R. Ferrari, and R. Hallberg, 2008: Parameterizing submesoscale physics in global climate models. CLIVAR Exchanges, No. 13, International CLIVAR Project Office, Southampton, United Kingdom, 3-5.

Gargett, A., and J. Wells, 2007: Langmuir turbulence in shallow water. Part 1. Observations. J. Fluid Mech., 576, 27-61, https:// doi.org/10.1017/S0022112006004575.

— , and C. Grosch, 2014: Turbulence process domination under the combined forcings of wind stress, the Langmuir vortex force, and surface cooling. J. Phys. Oceanogr., 44, 44-67, https://doi.org/10.1175/JPO-D-13-021.1.

— J. Jells, A. E. Tejada-Martínez, and C. E. Grosch, 2004: Langmuir supercells: A mechanism for sediment resuspension and transport in shallow seas. Science, 306, 1925-1928, https:// doi.org/10.1126/science.1100849.

_ D. Davidge, and J. Wells, 2014: Anatomy of a Langmuir supercell event. J. Mar. Res., 72, 127-163, https://doi.org/10.1357/ 002224014814901976.

Gerbi, P., J. Trowbridge, E. Terry, A. Plueddemann, and T. Kukulka, 2009: Observations of turbulence in the ocean surface boundary layer: Energetics and transport. J. Phys. Oceanogr., 39, 1077-1096, https://doi.org/10.1175/2008JPO4044.1.

Germano, M., U. Piomelli, P. Moin, and W. Cabot, 1991: A dynamic subgrid-scale eddy viscosity model. Phys. Fluids, 3A, 1760-1765, https://doi.org/10.1063/1.857955.

Golshan, R., A. E. Tejada-Martínez, M. Juha, and Y. Bazilevs, 2017: LES and RANS simulation of wind- and wave-forced oceanic turbulent boundary layers in shallow water with wall modeling. Comput. Fluids, 142, 96-108, https://doi.org/10.1016/ j.compfluid.2016.05.016.

Graham, A., and A. Hall, 1997: The horizontal distribution of bubbles in a shallow sea. Cont. Shelf Res., 17, 1051-1082, https://doi.org/10.1016/S0278-4343(97)00006-X.

Graham, J., and C. Meneveau, 2012: Modeling turbulent flow over fractal trees using renormalized numerical simulation: Alternate formulations and numerical experiments. Phys. Fluids, 24, 125105, https://doi.org/10.1063/1.4772074.

Grant, A., and S. Belcher, 2009: Characteristics of Langmuir turbulence in the ocean mixed layer. J. Phys. Oceanogr., 39, 1871-1887, https://doi.org/10.1175/2009JPO4119.1.

Grosch, C., and A. Gargett, 2016: Why do LES of Langmuir supercells not include rotation? J. Phys. Oceanogr., 46, 3595-3597, https://doi.org/10.1175/JPO-D-16-0092.1.

Haller, G., 2005: An objective definition of a vortex. J. Fluid Mech., 525, 1-26, https://doi.org/10.1017/S0022112004002526.

Harcourt, R., 2013: A second-moment closure model of Langmuir turbulence. J. Phys. Oceanogr., 43, 673-697, https://doi.org/ 10.1175/JPO-D-12-0105.1. 
— turbulence in pure wind seas. J. Phys. Oceanogr., 38, 1542-1562, https://doi.org/10.1175/2007JPO3842.1.

Harris, G., and J. Lott, 1973: Observations of Langmuir circulations in Lake Ontario. Limnol. Oceanogr., 18, 584-589, https:// doi.org/10.4319/lo.1973.18.4.0584.

Hinze, J., 1967: Secondary currents in wall turbulence. Phys. Fluids, 10 (Suppl.), S122-S125, https://doi.org/10.1063/1.1762429.

Hunt, J., A. Wray, and P. Moin, 1988: Eddies, stream, and convergence zones in turbulent flows. Proc. Center for Turbulence Research Summer Program, Stanford, CA, Stanford University, 193-208.

Hunter, R., and G. Hill, 1980: Near-shore current pattern off south Texas: An interpretation from aerial photographs. Remote Sens. Environ., 10, 115-134, https://doi.org/10.1016/0034-4257(80)90010-3.

Ichiye, T., 1965: Diffusion experiments in coastal waters using dye techniques. Proc. Symp. Diffusion in Oceans and Fresh Waters, Palisades, NY, Lamont Geological Observatory, 54-67.

Jeong, J., and F. Hussain, 1995: On the identification of a vortex. J. Fluid Mech., 285, 69-94, https://doi.org/10.1017/ S0022112095000462.

Kantha, L., and C. Clayson, 2004: On the effect of surface gravity waves on mixing in the oceanic mixed layer. Ocean Modell., 6 , 101-124, https://doi.org/10.1016/S1463-5003(02)00062-8.

Katz, B., R. Gerard, and M. Costin, 1965: Responses of dye tracers to sea surface conditions. J. Geophys. Res., 70, 5505-5513, https://doi.org/10.1029/JZ070i022p05505.

Kenney, B., 1977: An experimental investigation of the fluctuating currents responsible for the generation of windrows. Ph.D. thesis, University of Waterloo, $163 \mathrm{pp}$.

Kukulka, T., and P. Sullivan, 2013: Inhibited upper ocean restratification in nonequilibrium swell conditions. Geophys. Res. Lett., 40, 3672-3676, https://doi.org/10.1002/grl.50708.

— , and K. Brunner, 2015: Passive buoyant tracers in the ocean surface boundary layer: 1 . Influence of equilibrium windwaves on vertical distributions. J. Geophys. Res. Oceans, 120, 3837-3858, https://doi.org/10.1002/2014JC010487.

, and R. Harcourt, 2017: Influence of Stokes drift decay scale on Langmuir turbulence. J. Phys. Oceanogr., 47, 1637-1656, https://doi.org/10.1175/JPO-D-16-0244.1.

— A. Aleuddemann, J. Trowbridge, and P. Sullivan, 2009: Significance of Langmuir circulation in upper ocean mixing: Comparison of observations and simulations. Geophys. Res. Lett., 36, L10603, https://doi.org/10.1029/2009GL037620.

,,--- , and -2011 : The influence of crosswind tidal currents on Langmuir circulation in a shallow ocean. J. Geophys. Res., 116, C08005, https://doi.org/10.1029/2011JC006971.

- - - , and P. Sullivan, 2012: Nonlocal transport due to Langmuir circulation in a coastal ocean. J. Geophys. Res., 117, C12007, https://doi.org/10.1029/2012JC008340.

Langmuir, I., 1938: Surface motion of water induced by wind. Science, 87, 119-123, https://doi.org/10.1126/science.87.2250.119.

Lehr, W., and D. Beatty, 2000: The relation of Langmuir circulation processes to the standard oil spill spreading, dispersion and transport algorithms. Spill Sci. Technol. Bull., 6, 247-253, https://doi.org/10.1016/S1353-2561(01)00043-3.

Leibovich, S., 1977a: On the evolution of the system of wind drift currents and Langmuir circulations in the ocean. Part 1. Theory and the averaged current. J. Fluid Mech., 79, 715-743, https://doi.org/10.1017/S002211207700041X.

1977b: Convective instability of stably stratified water in the ocean. J. Fluid Mech., 82, 561-585, https://doi.org/10.1017/ S0022112077000846.
- 1983: The form and dynamics of Langmuir circulations. Annu. Rev. Fluid Mech., 15, 391-427, https://doi.org/10.1146/ annurev.fl.15.010183.002135.

Li, M., and C. Garrett, 1993: Cell merging and the jet/downwelling ratio in Langmuir circulation. J. Mar. Res., 51, 737-769, https:// doi.org/10.1357/0022240933223945.

— circulation. J. Phys. Oceanogr., 27, 121-132, https://doi.org/ 10.1175/1520-0485(1997)027<0121:MLDDTL>2.0.CO;2.

,$- \ldots$, and E. Skyllingstad, 2005: A regime diagram for classifying turbulent large eddies in the upper ocean. Deep-Sea Res. I, 52, 259-278, https://doi.org/10.1016/j.dsr.2004.09.004.

- J. Trowbridge, and W. Geyer, 2008: Asymmetric tidal mixing due to the horizontal density gradient. J. Phys. Oceanogr., 38, 418-434, https://doi.org/10.1175/2007JPO3372.1.

—, S. Radhakrishnan, U. Piomelli, and W. R. Geyer, 2010: Large-eddy simulation of the tidal-cycle variations of an estuarine boundary layer. J. Geophys. Res., 115, C08003, https:// doi.org/10.1029/2009JC005702.

Li, S., M. Li, G. Gerbi, and J.-B. Song, 2013: Roles of breaking waves and Langmuir circulation in the surface boundary layer of a coastal ocean. J. Geophys. Res. Oceans, 118, 5173-5187, https://doi.org/10.1002/jgrc.20387.

Maratos, A., 1971: Study of the near shore surface characteristics of windrows and Langmuir circulation in Monterey Bay. M.S. thesis, Dept. of Oceanography, Naval Postgraduate School, $155 \mathrm{pp}$.

Marmorino, G., G. Smith, and G. Lindemann, 2005: Infrared imagery of large-aspect-ratio Langmuir circulation. Cont. Shelf Res., 25, 1-6, https://doi.org/10.1016/j.csr.2004.08.002.

Martinat, G., Y. Xu, C. Grosch, and A. E. Tejada-Martínez, 2011: LES of turbulent surface shear stress and pressure-gradientdriven flow on shallow continental shelves. Ocean Dyn., 61, 1369-1390, https://doi.org/10.1007/s10236-011-0450-3.

Mason, P., 1994: Large-eddy simulation: A critical review of the technique. Quart. J. Roy. Meteor. Soc., 120, 1-26, https:// doi.org/10.1002/qj.49712051503.

McWilliams, J., and P. Sullivan, 2000: Vertical mixing by Langmuir circulations. Spill Sci. Technol. Bull., 6, 225-237, https:// doi.org/10.1016/S1353-2561(01)00041-X.

—,- , and C.-H. Moeng, 1997: Langmuir turbulence in the ocean. J. Fluid Mech., 334, 1-30, https://doi.org/10.1017/ S0022112096004375.

Meneveau, C., and J. Katz, 2000: Scale-invariance and turbulence models for large-eddy simulation. Annu. Rev. Fluid Mech., 32, 1-32, https://doi.org/10.1146/annurev.fluid.32.1.1.

Myer, G., 1969: A field investigation of Langmuir circulations. Proc. 12th Conf. on Great Lakes Research, Ann Arbor, MI, International Association of Great Lakes Research, 625-663.

Orszag, S., 1970: Transform method for calculation of vector coupled sums: Application to the spectral form of the vorticity equation. J. Atmos. Sci., 27, 890-895, https://doi.org/10.1175/ 1520-0469(1970)027<0890:TMFTCO >2.0.CO;2.

Owen, J. R., 1966: Small-scale, horizontal vortices in the surface layer of the sea. J. Mar. Res., 24, 56-65.

Oye, L., T. Ezer, and H. C. Lee, 2005: Loop current, rings and related circulation in the Gulf of Mexico: A review of numerical models and future challenges. Circulation in the Gulf of Mexico: Observations and Models, Geophys. Monogr., Vol. 161, Amer. Geophys. Union, 31-56.

Phillips, O., 1966: The Dynamics of the Upper Ocean. Cambridge University Press, $261 \mathrm{pp}$. 
Piomelli, U., and E. Balaras, 2002: Wall-layer models for large eddy simulations. Annu. Rev. Fluid Mech., 34, 349-374, https:// doi.org/10.1146/annurev.fluid.34.082901.144919.

Polton, J., and S. Belcher, 2007: Langmuir turbulence and deeply penetrating jets in an unstratified mixed layer. J. Geophys Res., 112, C09020, https://doi.org/10.1029/2007JC004205.

Scott, J., G. Myer, R. Stewart, and E. Walther, 1969: On the mechanism of Langmuir circulations and their role in epilimnion mixing. Limnol. Oceanogr., 14, 493-503, https://doi.org/ 10.4319/lo.1969.14.4.0493.

Scully, M., A. Fisher, S. Suttles, L. Sanford, and W. Boicourt, 2015: Characterization and modulation of Langmuir circulation in Chesapeake Bay. J. Phys. Oceanogr., 45, 2621-2638, https:// doi.org/10.1175/JPO-D-14-0239.1.

Sherwood, C., J. Lacey, and G. Voulgaris, 2006: Shear velocity estimates on the inner shelf off Grays Harbor, Washington, USA. Cont. Shelf Res., 26, 1995-2018, https://doi.org/10.1016/j.csr.2006.07.025.

Shrestha, K., J. Kuehl, and W. Anderson, 2017a: Impact of tidal current-wind-wave obliquity on the form and orientation of coastal Langmuir circulations. 2017 Fall Meeting, New Orleans, LA, Amer. Geophys. Union, Abstract NG21A-0124, https:// agu.confex.com/agu/fm17/meetingapp.cgi/Paper/221838.

- — - and — 2017b: Understanding the dimensional and mechanical properties of coastal Langmuir circulations. 70th Annual Meeting of the APS Division of Fluid Dynamics, Denver, CO, American Physical Society, E39.00001, http:// meetings.aps.org/link/BAPS.2017.DFD.E39.1.

_ - W. Anderson, and J. Kuehl, 2018: Coastal Langmuir circulations under misaligned currents, wind, and wave forcing. Gulf of Mexico Oil Spill and Ecosystem Science Conf., New Orleans, LA, Harte Research Institute for Gulf of Mexico Studies, RSP002-03, https://event.crowdcompass.com/gomoses2018/activity/ z0aMa6y5RX.

Sinha, N., A. E. Tejada-Martínez, and C. Akan, 2015: Toward a K-profile parameterization of Langmuir turbulence in shallow coastal shelves. J. Phys. Oceanogr., 45, 2869-2895, https:// doi.org/10.1175/JPO-D-14-0158.1.

Smith, J., 1992: Observed growth of Langmuir circulation. J. Geophys. Res., 97, 5651-5664, https://doi.org/10.1029/ 91JC03118.

_ R. Pinkel, and R. Weller, 1987: Velocity structure in the mixed layer during MILDEX. J. Phys. Oceanogr., 17, 425-439, https:// doi.org/10.1175/1520-0485(1987)017<0425:VSITML>2.0.CO;2.

Smith, W. N., J. Katz, and T. Osborn, 2005: On the structure of turbulence in the bottom boundary layer of the coastal ocean. J. Phys. Oceanogr., 35, 72-93, https://doi.org/10.1175/JPO-2673.1.

Smyth, W., E. Skyllingstad, G. Crawford, and H. Wijesekera, 2002: Nonlocal fluxes and Stokes drift effects in the K-profile parameterization. Ocean Dyn., 52, 104-115, https://doi.org/ 10.1007/s10236-002-0012-9.

Sternberg, W., 1968: Friction factors in tidal channels with differing bed roughness. Mar. Geol., 6, 243-260, https://doi.org/10.1016/ 0025-3227(68)90033-9.

Sullivan, P., and J. Williams, 2010: Dynamics of winds and currents coupled to surface waves. Annu. Rev. Fluid Mech., 42, 19-42, https://doi.org/10.1146/annurev-fluid-121108-145541.
Sutcliffe, W., E. Baylor, and D. Menzel, 1963: Sea surface chemistry and Langmuir circulations. Deep-Sea Res., 10, 233-243.

Tejada-Martínez, A., and C. Grosch, 2007: Langmuir turbulence in shallow water. Part 2. Large-eddy simulation. J. Fluid Mech., 576, 63-108, https://doi.org/10.1017/S0022112006004587.

A. Gargett, J. Polton, J. Smith, and J. MacKinnon, 2009: A hybrid spectral/finite-difference large-eddy simulator of turbulent processes in the upper ocean. Ocean Modell., 30, 115-142, https://doi.org/10.1016/j.ocemod.2009.06.008.

,,-- N. Sinha, C. Akan, and G. Martinat, 2012: Disruption of the bottom log layer in large-eddy simulations of full-depth Langmuir circulation. J. Fluid Mech., 699, 79-93, https:// doi.org/10.1017/jfm.2012.84.

, C. Akan, N. S. C. Grosch, and G. Martinat, 2013: Surface dynamics in LES of full-depth Langmuir circulation in shallow water. Phys. Scr., 2013, 014008, https://doi.org/10.1088/00318949/2013/T155/014008.

Thorpe, S., 2000: Langmuir circulation and the dispersion of oil spills in shallow seas. Spill Sci. Technol. Bull., 6, 213-223, https://doi.org/10.1016/S1353-2561(01)00040-8.

_, 2004: Langmuir circulation. Annu. Rev. Fluid Mech., 36, 5579, https://doi.org/10.1146/annurev.fluid.36.052203.071431.

_ , and A. Hall, 1982: Observations of the thermal structure of Langmuir circulation. J. Fluid Mech., 114, 237-250, https:// doi.org/10.1017/S0022112082000123.

Van Straaten, L., 1950: Periodic patterns of rippled and smooth areas on water surfaces induced by wind action. Proc. K. Ned. Akad. Wet., 53B, 1217-1227.

Walker, R., A. E. Tejada-Martínez, and E. Chester, 2016: Largeeddy simulation of a coastal ocean under the combined effects of surface heat fluxes and full-depth Langmuir circulation. J. Phys. Oceanogr., 46, 2411-2436, https://doi.org/10.1175/ JPO-D-15-0168.1.

Weller, R., and J. Price, 1988: Langmuir circulation within the oceanic mixed layer. Deep-Sea Res., 35, 711-747, https:// doi.org/10.1016/0198-0149(88)90027-1.

Willingham, D., W. Anderson, K. T. Christensen, and J. Barros, 2013: Turbulent boundary layer flow over transverse aerodynamic roughness transitions: Induced mixing and flow characterization. Phys. Fluids, 26, 025111, https://doi.org/ 10.1063/1.4864105.

Yang, D., B. Chen, M. Chamecki, and C. Meneveau, 2015: Oil plumes and dispersion in Langmuir, upper-ocean turbulence: Large-eddy simulations and K-profile parameterization. J. Geophys. Res. Oceans, 120, 4729-4759, https://doi.org/ 10.1002/2014JC010542.

Yang, J., and W. Anderson, 2018: Turbulent channel flow over surfaces with variable spanwise heterogeneity: Establishing conditions for outer-layer similarity. Flow Turbul. Combust., 100, 1-17, https://doi.org/10.1007/s10494-017-9839-5.

Zedel, L., and D. Farmer, 1991: Organised structures in subsurface bubble cloud: Langmuir circulation in the open ocean. J. Geophys. Res., 96, 8889-8900, https://doi.org/10.1029/ 91JC00189.

Zikanov, O., D. Slinn, and M. Dhanak, 2003: Large-eddy simulations of the wind-induced turbulent Ekman layer. J. Fluid Mech., 495, 343-368, https://doi.org/10.1017/S0022112003006244. 